\title{
Dynamics of a hyperbolic system that applies at the onset of the oscillatory instability
}

\author{
Carlos Martel and José $M$ Vega \\ ETSI Aeronáuticos, Universidad Politécnica de Madrid, Plaza Cardenal Cisneros, 328040
}

Madrid, Spain

\begin{abstract}
A real hyperbolic system is considered that applies near the onset of the oscillatory instability in large spatial domains. The validity of that system requires that some intermediate scales (large compared with the basic wavelength of the unstable modes but small compared with the size of the system) remain inhibited; that condition is analysed in some detail. The dynamics associated with the hyperbolic system is fully analysed to conclude that it is very simple if the coefficient of the cross-nonlinearity is such that $-1<\alpha \leqslant 1$, while the system exhibits increasing complexity (including period-doubling sequences, quasiperiodic transitions, crises) as the bifurcation parameter grows if $\alpha>1$ if $\alpha \leqslant-1$ then the system behaves subcritically. Our results are seen to compare well, both qualitatively and quantitatively, with the experimentally obtained ones for the oscillatory instability of straight rolls in pure Rayleigh-Bénard convection.
\end{abstract}

AMS classification scheme numbers: 35B32, 35B35, 35B40, 76E30

\section{Introduction}

This paper is concerned with the following hyperbolic system

$$
u_{f}-u_{x}=2 u(\lambda-u-\alpha v) \quad v_{f}+v_{x}=2 v(\lambda-v-\alpha u)
$$

in $-\frac{1}{2}<x<\frac{1}{2}$ and $t \geqslant 0$. with boundary conditions

$$
v=R u \quad \text { at } x=-\frac{1}{2} \quad \text { and } \quad u=R v \quad \text { at } x=\frac{1}{2}
$$

and initial conditions

$$
u=u_{0}(x)>0 \quad v=v_{0}(x)>0 \quad \text { in }-\frac{1}{2} \leqslant x \leqslant \frac{1}{2}
$$

that are assumed to satisfy the compatibility conditions

$$
\begin{array}{ll}
v_{0}^{\prime}+R u_{0}^{\prime}=2(\alpha-1)(R-1) R u_{0}^{2} & \text { at } x=-\frac{1}{2} \\
u_{0}^{\prime}+R v_{0}^{\prime}=2(\alpha-1)(1-R) R v_{0}^{2} & \text { at } x=\frac{1}{2} .
\end{array}
$$

These conditions prevent the formation of singularities that would propagate along the characteristics of (1.1) (see, e.g. [1]). The problem depends on the bifurcation parameter $\lambda$, on the coupling parameter $\alpha$ and on the reflection coefficient $R>0$. 
Let us now briefly explain how this real hyperbolic system is obtained as a sub-model of the following coupled, complex Ginzburg-Landau equations

$$
\begin{aligned}
& A_{T}=c A_{X X}+b A_{X}+d \mu A-\left(e_{1}|A|^{2}+e_{2}|B|^{2}\right) A \\
& B_{T}=c B_{X X}-b B_{X}+d \mu B-\left(e_{1}|B|^{2}+e_{2}|A|^{2}\right) B
\end{aligned}
$$

where $A$ and $B$ are the complex amplitudes of a pair of counter-propagating wavetrains that appear at the onset of the so-called oscillatorv instability on a dissipative plysical system that is invariant under spatial translation and reflection. see [2]. The state variable $w$ of the physical system may be written in terms of those wavetrains as

$w=A(X, T) W_{0}^{+} \exp (\mathrm{i} \Omega T+\mathrm{i} k X)+B(X, T) W_{0}^{-} \exp (\mathrm{i} \Omega 2 t-\mathrm{i} k X)+$ c.c. + HOT

where $\Omega>0, k$ and $W_{0}^{ \pm}$are the frequency, the wavenumber and a pair of eigenvectors associated with the marginally unstable counter-propagating modes, and c.c. and HOT stand for the complex conjugate and higher-order terms respectively. Equations (1.6) and (1.7) are derived by a weakly nonlinear analysis whose consistency requires the complex amplitudes $A$ and $B$ to be small and to depend weakly on space and time. and the bifurcation parameter $\mu$ to be small. i.e. essentially that

$|\mu| \ll 1 \quad\left|A_{X X}\right| \ll\left|A_{X}\right| \ll|A| \ll 1 \quad\left|B_{X X}\right| \ll\left|B_{X}\right| \ll|B| \ll 1$.

The real coefficient $b$ (i.e. the group velocity) and the real and imaginary parts of the complex coefficients $c, d, e_{1}$ and $e_{2}$ (accounting for diffusion. dispersion. linear growth and nonlinearity) are assumed to be bounded away from zero. as is the case generically (see remark (b), in section 7). In addition. it is assumed that

$$
c+\bar{c}>0 \quad e_{1}+\bar{e}_{1}>0 \text { and } \quad d+\bar{d}>0
$$

where the overbar stands hereafter for the complex conjugate. If the first condition does not hold then either: (i) a fully nonlinear description of the underlying physical system must be considered (if $|c+\bar{c}| \sim 1$ ) or (ii) higher-order derivatives must be added to (1.6) and (1.7) to obtain the correct normal form (if $|c+\bar{c}| \ll 1$ ); the second condition comes from the requirement that the bifurcation is supercritical (supercriticality will also require that $e_{1}+\bar{e}_{1}>-\left(e_{2}+\bar{e}_{2}\right)$, as it will be seen below). The last condition always holds if the sign of the bifurcation parameter is appropriately chosen (for the instability to appear as $\mu$ increases).

If equations (1.6) and (1.7) are considered in a large but finite interval, $-L / 2<X<$ $L / 2$, with

$$
L \gg 1
$$

then four boundary conditions must be imposed. Two of them were first introduced by Cross [3]

$$
B=r A \quad \text { at } X=-L / 2 \quad A=r B \quad \text { at } X=L / 2
$$

and account for the linear reflection of the wavetrains at the end-walls; they depend only on the complex reflection coefficient $r \neq 0$. The other two boundary conditions are nonlinear if $e_{1} \neq e_{2}$ and $|r| \neq 1$,

$$
\begin{array}{ll}
b\left(B_{X}+r A_{X}\right)=\left(e_{2}-e_{1}\right) r\left(|r|^{2}-1\right) A|A|^{2} & \text { at } X=-L / 2 \\
b\left(A_{X}+r B_{X}\right)=\left(e_{2}-e_{1}\right) r\left(1-|r|^{2}\right) B|B|^{2} & \text { at } X=L / 2
\end{array}
$$

and were first calculated in [4], where conditions (1.12) were also justified with great generality. In addition to being the correct boundary conditions, any other boundary 
conditions different from (1.13) and (1.14) would lead to inconsistencies, as it will be explained below (remark (c) in section 2).

The model posed by (1.6). (1.7), (1.12)-(1.14) applies to a large variety of plysical problems. including at least binary fluid convection, isothermal double-diffusive convection, pure Rayleigh-Benard convection (as a secondary instability of rolls). (spiral waves in) the Taylor-Couette system. (transversal waves in) several thermocapillary flows. electrodynamic convection in nematic liquid crystals and several combustion systems (see $[4,5]$ ).

Note that, according to (1.9). equations (1.6) and (1.7) contain terms that are not of the same order. This fact allows further simplifications in two distinguished limits (or regimes), that are briefly described now. To this end, first note that the basic steady state of (1.6), (1.7), (1.12)-(1.14) becomes unstable as

$$
\mu>\mu_{c} \equiv-2 L^{-1} b(d+\bar{d})^{-1} \log |r|+\mathrm{O}\left(L^{-2}\right) .
$$

This shift in the instability limit is due to the presence of non-perfectly reflecting boundaries, as first pointed out by Cross [3] to explain some experimental results.

The first distinguished regime corresponds to

$$
\left|\mu-\mu_{c}\right| \sim L^{-2} \quad|A| \sim|B| \sim L^{-1}
$$

and its analysis requires considering one spatial scale, $X \sim L$, and two time scales, $T \sim L$ and $T \sim L^{2}$, to obtain a non-local, complex Ginzburg-Landau equation for the evolution of the wavetrains in the slowest time scale. In the particular case of perfectly reflecting boundaries, $|r|=1$, the first non-local equation seems to have been obtained by Chikwendu and Kevorkian [6] in a related setting, and by Knobloch and De Luca [7] and AlvarezPereira and Vega [8] in the analysis of the oscillatory instability of steady states in one dimension and of travelling wavefronts in two dimensions respectively; see also [9] for a rigorous derivation of the equation, [10] for the analysis of inertial manifolds and related global-dynamics properties, and [11] for related conservative equations. The derivation of this non-local equation in the (much more subtle) case $|r| \neq 1$ is given in [4]. The equation has an intrinsic interest and exhibits a quite rich variety of complex dynamic behaviours resulting from the interplay of the modulational instability and end-wall effects, see $[4,12,13]$. Nevertheless, this limit applies only in a quite narrow region near the instability limit (see (1.16)) and thus it is less interesting from the practical point of view than the second limit, that is now considered.

In the second distinguished regime. corresponding to

$$
\left|\mu-\mu_{c}\right| \sim|\mu| \sim L^{-1} \quad|A| \sim|B| \sim L^{-1 / 2}
$$

we rescale the space and time variables and the complex amplitudes as

$$
\begin{aligned}
& x=X / L \quad t=|b| T / L \\
& \left(A_{1}, B_{1}\right)=\sqrt{\left(e_{1}+\bar{e}_{1}\right) L /(2|b|)} \exp [(d-\bar{d}) T / 2](A, B)
\end{aligned}
$$

to rewrite $(1.6),(1.7)$ and $(1.12)-(1.14)$ as

$$
\begin{aligned}
& A_{1 t}=\varepsilon\left(1+\mathrm{i} \alpha_{1}\right) A_{1 x x}+(b /|b|) A_{1 x}+\left[\lambda-\left(1+\mathrm{i} \alpha_{2}\right)\left|A_{1}\right|^{2}-\left(\alpha+\mathrm{i} \alpha_{3}\right)\left|B_{1}\right|^{2}\right] A_{1} \\
& B_{1 t}=\varepsilon\left(1+\mathrm{i} \alpha_{1}\right) B_{1 x x}-(b /|b|) B_{1 x}+\left[\lambda-\left(1+\mathrm{i} \alpha_{2}\right)\left|B_{1}\right|^{2}-\left(\alpha+\mathrm{i} \alpha_{3}\right)\left|A_{1}\right|^{2}\right] B_{1} \\
& B_{1}=\rho \mathrm{e}^{\mid \alpha_{4}} A_{1} \quad \text { at } x=-\frac{1}{2} \quad A_{1}=\rho \mathrm{e}^{\mathrm{i} \alpha_{4}} B_{1} \quad \text { at } x=\frac{1}{2} \\
& B_{1 x}+\rho \mathrm{e}^{\mathrm{i} \alpha_{4}} A_{1, x}=\rho \mathrm{e}^{\mathrm{i} \alpha_{4}}\left(1-\rho^{2}\right)(b /|b|)\left[1-\alpha+\mathrm{i}\left(\alpha_{2}-\alpha_{3}\right)\right] A_{1}\left|A_{1}\right|^{2} \quad \text { at } x=-\frac{1}{2} \\
& A_{1 x}+\rho \mathrm{e}^{\mid \alpha_{4}} B_{1 x}=\rho \mathrm{e}^{\mathrm{i} \alpha_{4}}\left(\rho^{2}-\mathbf{l}\right)(b /|b|)\left[1-\alpha+\mathrm{i}\left(\alpha_{2}-\alpha_{3}\right)\right] B_{1}\left|B_{1}\right|^{2} \quad \text { at } x=\frac{1}{2}
\end{aligned}
$$


where the rescaled bifurcation parameter, the real coefficients $\alpha_{1}, \ldots, \alpha_{4}, \alpha$ and $\rho>0$, and the small parameter $\varepsilon>0$ are defined as

$$
\begin{array}{lcc}
1+\mathrm{i} \alpha_{1}=2 c /(c+\bar{c}) & 1+\mathrm{i} \alpha_{2}=2 e_{1} /\left(e_{1}+\bar{e}_{1}\right) & \alpha+\mathrm{i} \alpha_{3}=2 e_{2} /\left(e_{1}+\bar{e}_{1}\right) \\
\lambda=(d+\bar{d}) \mu L /(2|b|) & r=\rho \mathrm{e}^{\mathrm{i} \alpha_{4}} \quad \varepsilon=(c+\bar{c}) /(2|b| L) .
\end{array}
$$

Then we only need to neglect the $O(\varepsilon)$ terms in $(1.19)$ and $(1.20)$ and to define the variables $u$ and $v$ and the parameter $R>0$ as

$$
\begin{array}{llll}
u=\left|A_{1}\right|^{2} & v=\left|B_{1}\right|^{2} & R=\rho^{2} & \text { if } b>0 \\
u=\left|B_{1}\right|^{2} & v=\left|A_{1}\right|^{2} & R=1 / \rho^{2} & \text { if } b<0
\end{array}
$$

to obtain (1.1) and (1.2) from (1.19) and (1.21).

This paper is organized as follows. In section 2 we shall give some comments on this second regime and some preliminary results concerning the real hyperbolic system (1.1)(1.3). The steady states of (1.1) and (1.2) and their linear stability will be considered in section 3 . while the global dynamics will be numerically analysed in section 4 by means of dynamical systems techniques. The validity of (1.1)-(1.3) as an approximation of (1.19)(1.23) (as $\varepsilon \rightarrow 0$ ) will be analysed in section 5. Finally, in section 6 some qualitative and quantitative comparisons with experiments will be made, and in section 7 some concluding remarks will be drawn.

\section{Some additional preliminaries}

The derivation of the real hyperbolic system (1.1)-(1.3) from the rescaled normal form (1.19)-(1.23) (as $\varepsilon \rightarrow 0$ ) exhibits several subtleties that should not be ignored. Some remarks on this derivation and the physical interpretation of the solutions of $(1.1)-(1.3)$ are now in order.

(a) Those terms accounting for diffusion and dispersion in (1.19) and (1.20) have been ignored. We must assume that these terms are initially small and check that they remain small as time proceeds. This is a stability question; namely, we must ascertain that small perturbations. depending on intermediate scales, with a characteristic length of the order of $\sqrt{\varepsilon}$ (such that the neglected terms are of the order of nonlinear terms) are damped out as time proceeds. According to (1.18), the characteristic size of these scales is in between of the basic $O(\varepsilon)$ wavelength of the counter-propagating wavetrains and the $O(I)$ length of the domain. We shall study these intermediate scales in section 5 .

(b) If, according to (1.25), the phases of $A_{1}$ and $B_{1}$ are defined as $A_{1}=\sqrt{u} \mathrm{e}^{\mathrm{i} \theta}$ and $B_{1}=\sqrt{v} \mathrm{e}^{\mathrm{i} \varphi}$ if $b>0$, and $A_{1}=\sqrt{v} \mathrm{e}^{\mathrm{i} \varphi}$ and $B_{1}=\sqrt{u} \mathrm{e}^{\mathrm{i} \theta}$ if $b<0$, then the following equations for $\theta$ and $\varphi$ are obtained from (1.19)-(1.21) in first approximation

$\theta_{t}-\theta_{x}+\alpha_{2} u+\alpha_{3} v=\varphi_{t}+\varphi_{x}+\alpha_{2} v+\alpha_{3} u=0$

$\varphi=\theta+\alpha_{4} b /|b| \quad$ at $x=-\frac{1}{2} \quad \theta=\varphi+\alpha_{4} b /|b| \quad$ at $\quad x=\frac{1}{2}$.

Once $u$ and $v$ have been calculated from (1.1)-(1.3) and inserted into (2.1) and (2.2) we obtain a linear problem whose solution depends on an arbitrary function (associated with the solution of the homogeneous problem) that evolves in a still slower scale. When considering higher-order terms, a nonlinear equation may be obtained for this evolution that exhibits chaotic dynamics (corresponding to phase turbulence) for appropriate values of the parameters. That equation will be derived and analysed elsewhere. 
(c) The second pair of boundary conditions (1.22), (1.23) have not been used to derive the hyperbolic problem (1.1)-(1.3), and may be written as

$$
\begin{array}{ll}
v_{x}+R u_{x}=2(\alpha-1)(R-1) R u^{2} & \text { at } x=-\frac{1}{2} \\
u_{x}+R v_{x}=2(\alpha-1)(1-R) R v^{2} & \text { at } x=\frac{1}{2} .
\end{array}
$$

These conditions are precisely the compatibility conditions of the hyperbolic problem, that are assumed to hold initially (see (1.4) and (1.5)) and consequently they also hold for all $t>0$ (insert (1.1) into (2.3) and take into account that $v_{t}=R u_{t}$ at $x=-\frac{1}{2}$ and $u_{t}=R v_{t}$ at $x=\frac{1}{2}$, according to (1.2)). This fact is essential for the consistency of our weakly nonlinear description because. if the second pair of boundary conditions was not satisfied by the hyperbolic problem, then two boundary layers should be added near $x= \pm \frac{1}{2}$ to take care of them; in these boundary layers we would have $\varepsilon\left|A_{1 x x}\right| \sim\left|A_{1 x}\right|$ and/or $\varepsilon\left|B_{1 x x}\right| \sim\left|B_{1 x}\right|$ or. in the original variables, $\left|A_{X X}\right| \sim\left|A_{X}\right|$ and/or $\left|B_{X X}\right| \sim\left|B_{X}\right|$, and thus the consistency requirements (1.9) would be violated. Thus. the second pair of boundary conditions, (1.13), (1.14), are the natural ones, as anticipated above.

(d) Note that, according to $(1.25), u \geqslant 0$ and $v \geqslant 0$. In fact we have assumed that the initial conditions for $u$ and $v$ are strictly positive (see (1.3)) and thus $u>0$ and $v>0$ for all $t>0$ (see [14]). If instead the initial conditions (1.3) are allowed to vanish then the number of zeros of $u$ and $v$ in $-\frac{1}{2} \leqslant x \leqslant \frac{1}{2}$ remains constant as time proceeds (the zeros propagate along the characteristics); but this property is non-generic (a small perturbation on the initial conditions eliminates the zeros) and spurious as approximation of (1.19)(1.23) (the neglected second-order spatial derivatives preclude the zeros of $\left|A_{1}\right|^{2}$ and $\left|B_{1}\right|^{2}$ to remain as time proceeds).

(e) The reflection coefficient of the hyperbolic problem $R$ deserves some attention. Its relation with the original complex reflection coefficient $r$ is given by (1.25). Then even in the frequent case of absorbing boundaries, $\rho=|r|<1, R$ may be larger than 1 if the group velocity $b$ is negative (as in the Taylor-Couette system, see [15]). Then we shall pay the same attention below to the cases $R>1$ and $R<1$; the (non-generic without further restrictions) case of perfectly reflecting boundaries, $\rho=R=1$, will be also considered in some detail.

In order to plot the counter-propagating wavetrains associated with the solutions of (1.1), (1.2), we consider the state variable

$$
\begin{array}{ll}
w=\sqrt{u} \mathrm{e}^{\mathrm{i}(\Omega t+k x) L}+\sqrt{v} \mathrm{e}^{\mathrm{i}(\Omega t-k x) L}+c . c . & \text { if } R \leqslant 1 \\
w=\sqrt{v} \mathrm{e}^{\mathrm{i}(\Omega t+k x) L}+\sqrt{u} \mathrm{e}^{\mathrm{i}(\Omega t-k x) L}+\text { c.c. } & \text { if } R>1
\end{array}
$$

where we are taking into account (1.18) and (1.25) and for illustration we are considering particularly simple values of the coefficients of the amplitude equations (1.6). (1.7) and (1.19), (1.20) (i.e. $d-\bar{d}=\alpha_{2}=\alpha_{3}=\alpha_{4}=0$ ) and of the phases of the complex amplitudes (i.e. $\theta \equiv \varphi \equiv 0$, see (2.1). (2.2)). In addition. we are ignoring the scaling factor for the complex amplitudes in (1.18) and we are assuming the end-walls to be either purely reflecting or absorbing (as is frequently the case in practice) and thus we are taking the group velocity $b$ in (1.25) to be positive if $R \leqslant 1$ and negative if $R>1$, according to remark (e) above. The plots will be made for the following particular values of the scaled frequency $\Omega L$ and wavenumber $k L$

$$
\Omega L=k L=15 \pi \text {. }
$$

The problem (1.1)-(1.3) was analysed in [14] by means of comparison methods based on coupled sub- and supersolutions. In particular, the following global existence and stability results were proven (see figure 1 for illustration). 


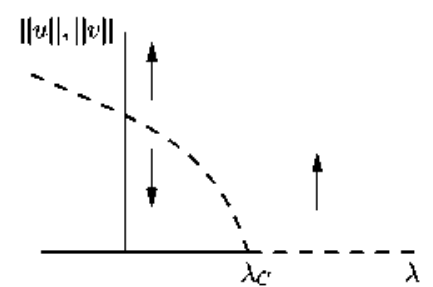

(a) $a<-1$

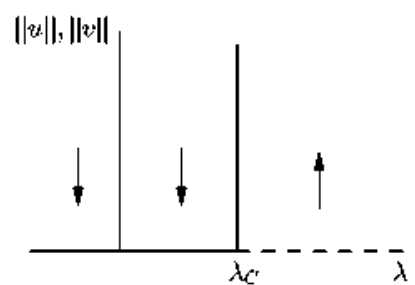

(b) $\alpha=-1$

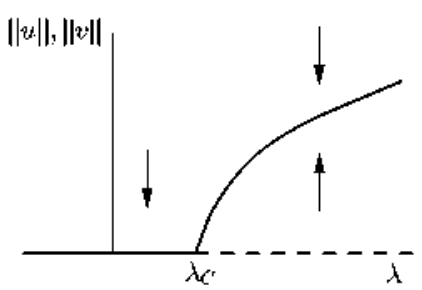

(c) $-1<\alpha \leq 1$

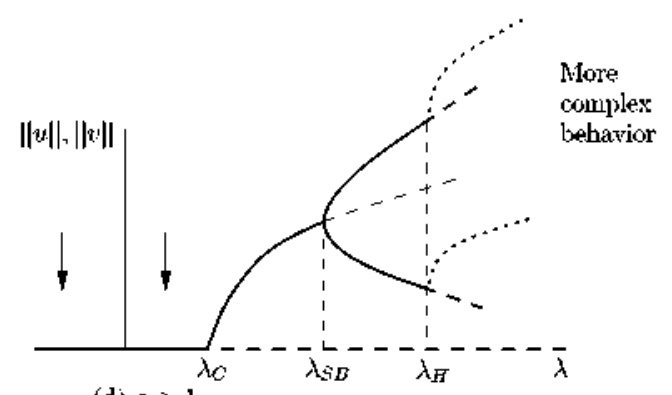

(d) $\alpha>1$

Figure 1. Sketches of the bifurcation diagrams for the hyperbolic system (1.1)-(1.3).

(i) If $u_{0}$ and $v_{0}$ are continuously differentiable and satisfy (1.4), (1.5) then the problem (1.1)-(1.3) has a unique, continuously differentiable solution such that $u>0$ and $v>0$ in $-\frac{1}{2} \leqslant x \leqslant \frac{1}{2}, 0 \leqslant t<T \leqslant \infty$, where $T$ is maximal in the following sense, either $T=\infty$ or $u+v$ becomes unbounded as $t \nearrow T$.

(ii) If $\alpha<-1, \lambda<\lambda_{C}=-(\log R) / 2$ and $u_{0}$ and $v_{0}$ are sufficiently small, then the solution of (1.1)-(1.3) converges uniformly to $u=v=0$ as $t \rightarrow \infty$. If $\alpha<-1$ and either $\lambda<\lambda_{\mathrm{C}}$ and $u_{0}$ and $v_{0}$ are sufficiently large, or $\lambda \geqslant \lambda_{\mathrm{C}}$ then the solution of (1.1)-(1.3) becomes unbounded at some finite $t=T$.

(iii) If $\alpha=-1$ and $\lambda \leqslant \lambda_{C}$ (resp. $\lambda>\lambda_{C}$ ) then every solution of (1.1)-(1.3) converges uniformly to $u=v=0$ (resp. becomes unbounded) as $t \rightarrow \infty$.

(iv) If $\alpha>-1$ then every solution of (1.1)-(1.3) is uniformly bounded in $-\frac{1}{2} \leqslant x \leqslant \frac{1}{3}$. $0 \leqslant t<\infty$. If, in addition, $\lambda \leqslant \lambda_{C}$, then every solution of (1.1)-(1.3) converges uniformly to $u=v=0$ as $t \rightarrow \infty$.

(v) If $-1<\alpha<1$ and $\lambda>\lambda_{C}$ then (1.1), (1.2) has a unique steady state such that $u_{s}>0$ and $v_{s}>0$ in $-\frac{1}{2} \leqslant x \leqslant \frac{1}{2}$, and every solution (1.1)-(1.3) converges uniformly to $\left(u_{s}, v_{s}\right)$ as $t \rightarrow \infty$.

Note that. according to properties (ii) and (iii). our weakly nonlinear description is subcritical if $\alpha<-1$ and critical if $\alpha=-1$ (figures $\mathbf{I}(a)$ and $(b)$ ) and thus supercriticality requires that $\alpha>-1$, or $e_{1}+\bar{e}_{1}>-\left(e_{2}+\bar{e}_{2}\right)$ according to (1.24), as anticipated right after equation (1.10). In the subcritical case a fully nonlinear description is required, while in the critical case higher-order (quintic, ...) terms must be added to (1.6), (1.7). Also, according to properties (iv) and (v), the dynamics of (1.1)-(1.3) is trivial (i.e. every solution converges to a steady state as $t \rightarrow \infty)$ if either $-1<\alpha<1$ or $\alpha \geqslant 1$ and $\lambda \leqslant \lambda_{C}$ (figures $\mathbf{l}(c)$ and $(d)$ ). If instead $\alpha \geqslant 1$ and $\lambda>\lambda_{C}$ then we only know that the solutions are bounded; the main objective of this paper is to analyse the dynamics of (1.1)-(1.3) in this case. In particular (see figure $1(d)$ ) we shall see that a branch of stable, symmetric (under the transformation $x \rightarrow-x, u \leftrightarrow v$ ) steady states bifurcates supercritically at $\lambda=\lambda_{C}$ from the basic steady state $u=v=0$. and exhibits a supercritical, pitchfork, stmmetry-breaking 
bifurcation at some $\lambda=\lambda_{\mathrm{SB}}>\lambda_{\mathrm{C}}$. The resulting branch of stable, non-symmetric steady states exhibits a Hopf bifurcation at some $\lambda=\lambda_{\mathrm{H}}>\lambda_{\mathrm{SB}}$. These three bifurcations are always present. The remaining part of the bifurcation diagram depends on the parameters $\alpha$ and $R$, but always exhibits additional transitions to chaotic attractors that alternate with non-chaotic ones. Among these additional bifurcations, the system exhibits period-doubling sequences, crises associated with collisions of periodic attractors through symmetry-gaining, intermittency and quasiperiodic transitions.

\section{The steady states and their stability}

The physically meaningful steady states of (1.1), (1.2) are given by

$$
\begin{aligned}
& \mathrm{d} u_{s} / \mathrm{d} x+2 u_{s}\left(\lambda-u_{s}-\alpha v_{s}\right)=\mathrm{d} v_{s} / \mathrm{d} x-2 v_{s}\left(\lambda-v_{s}-\alpha u_{s}\right)=0 \\
& v_{s}=R u_{s} \quad \text { at } x=-\frac{1}{2} \quad u_{s}=R v_{s} \quad \text { at } x=\frac{1}{2} \\
& u_{s} \geqslant 0 \quad v_{s} \geqslant 0 \quad \text { in }-\frac{1}{2} \leqslant x \leqslant \frac{1}{2} .
\end{aligned}
$$

The linear stability of a steady state $\left(u_{s}, v_{s}\right)$ is analysed by first linearizing $(1.1),(1.2)$ around $\left(u_{s}, v_{s}\right)$ and then introducing the ansatz $(u, v)=\left(u_{s}, v_{s}\right)+\mathrm{e}^{\omega t}(U(x), V(x))$, to obtain

$$
\begin{aligned}
& U^{\prime}-\left(\omega-2 \lambda+4 u_{s}+2 \alpha v_{s}\right) U-2 \alpha u_{s} V=0 \\
& V^{\prime}+\left(\omega-2 \lambda+4 v_{s}+2 \alpha u_{s}\right) V+2 \alpha v_{s} U=0 \\
& V=R U \quad \text { at } x=-\frac{1}{2} \quad U=R V \quad \text { at } x=\frac{1}{2} .
\end{aligned}
$$

The problem (3.1), (3.2) always possesses the trivial solution $u_{s} \equiv v_{s} \equiv 0$ : the associated eigenvalues and eigenfunctions of (3.4). (3.5) are $\omega=2\left(\lambda-\lambda_{C}\right)+i k \pi$ and $U(x)=(-1)^{k} V(-x)=C R^{x} \exp (\mathrm{i} k \pi x)$, where $C \neq 0$ is a constant, $k$ is an integer and

$$
\lambda_{\mathrm{C}}=-(\log R) / 2 \text {. }
$$

Thus, the trivial steady state is exponentially stable if $\lambda<\lambda_{C}$ and exponentially unstable if $\lambda>\lambda_{C}$. At $\lambda=\lambda_{C}$ there is a supercritical bifurcation to a new branch of steady states that is locally given by

$$
u_{s}(x)=v_{s}(-x)=\varepsilon R^{x}+\mathrm{O}\left(\varepsilon^{2}\right) \quad \lambda=\lambda_{C}+\varepsilon(\alpha+1)(R-1) /(\sqrt{R} \log R)+\mathrm{O}\left(\varepsilon^{2}\right)
$$

where $0 \leqslant \varepsilon \ll 1$ and $(R-1) / \log R$ must be replaced by 1 if $R=1$. Note that the bifurcated solutions are symmetric, i.e. they are invariant under the transformation

$$
x \rightarrow-x \quad u_{s} \leftrightarrow v_{s} .
$$

There are other branches of bifurcated and periodic solutions of (1.1). (1.2). associated with the eigenvalues $\omega=\mathrm{i} k \pi$. with $k \neq 0$, but those solutions cannot be accepted because they do not satisfy (I.3) (which implies that $u>0$ and $v>0$ for all $t>0$. as remarked at the end of section 1) because the solutions associated with these eigenvalues oscillate around $u=v=0$.

Let us now consider the remaining solutions of $(1.1)-(1.3)$, which are such that

$$
u_{s}>0 \quad \text { and } \quad v_{s}>0 \quad \text { in }-\frac{1}{2} \leqslant x \leqslant \frac{1}{2}
$$

because if either $u_{s}=0$ or $v_{s}=0$ at some $x$, then $u_{s}=v_{s}=0$ for all $x$, as obtained from uniqueness properties of the ODEs (3.1) and the boundary conditions (3.2). If $\alpha=-1,0$ or I. then the general solution of (3.1) may be found in closed form; the associated solutions 

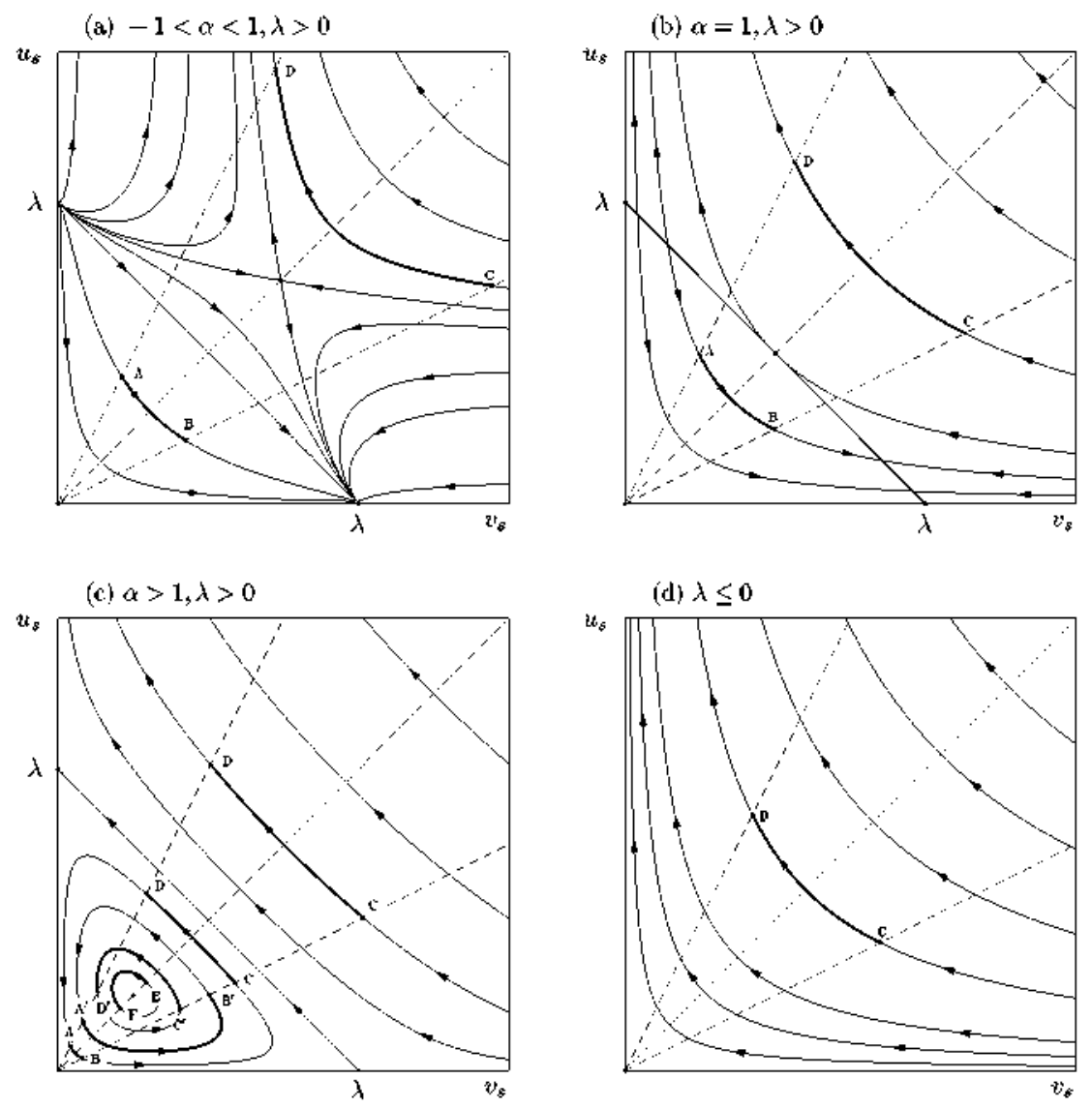

Figure 2. Phase portraits for the system (3.1). Heavy curves correspond to possible solutions and broken lines indicate the boundary conditions (3.2).

of (3.1). (3.2) are given in the appendix. If $\alpha>-1$ and $R=I$ then (3.1)-(3.3) possess the branch of spatially constant solutions

$$
u_{s}(x)=v_{s}(-x)=\lambda /(1+\alpha) .
$$

In the general case, $R \neq 1$, system (3.1) still has the first integral

$$
\left|u_{s}+v_{s}-\lambda\right|^{1-\alpha}=C u_{s} v_{s}
$$

where $C \geqslant 0$ is an arbitrary constant. Then (3.1)-(3.3) may be solved by quadratures, but this solution (is not quite useful because it) requires considering several cases that must be analysed separately. Instead. the bifurcation diagrams below will be obtained by means of a contimuation method (based on the ideas in, for example [16]) that is initiated on the approximate solutions $(3.7)$; the problem $(3.1),(3.2)$ is solved by a shooting method at each step in the continuation process.

In order to discuss the solutions of (3.1)-(3.3) we consider the phase portrait of (3.1). which is readily obtained (by means of the first integral (3.11)) to be as plotted in figure 2 , where only the first quadrant $u_{s} \geqslant 0, v_{s} \geqslant 0$ is considered; the arrows indicate increasing values of $x$. Note that the origin is always a critical point that behaves as a saddle. If $\lambda>0$ then system (3.I) also has the critical points $\left(u_{s}, v_{s}\right)=(\lambda /(\mathbf{I}+\alpha), \lambda /(\mathbf{I}+\alpha))$ (a saddle 
if $-1<\alpha<1$ and a centre if $\alpha>1),\left(u_{s}, v_{s}\right)=(\lambda, 0)$ and $\left(u_{s}, v_{s}\right)=(0, \lambda)$ (which are nodes if $-1<\alpha<1$ and saddles if $\alpha>1$ ); if $\alpha=1$, then all points in the line $u_{s}+v_{s}=\lambda$ are degenerate critical points. The solutions of (3.1)-(3.3) correspond to segments (plotted with heavy curves) of the orbits of (3.1) joining the straight lines $v_{s}=R u_{s}$ and $u_{s}=R v_{s}$ (which are plotted with broken curves) as $x$ varies from $-\frac{1}{2}$ to $\frac{1}{2}$ : note that this condition determines the actual solution. Several cases are now considered.

(a) If $-1<\alpha \leqslant 1$ then all solutions of (3.1)-(3.3) are symmetric and correspond (in figures $2(a)$. $(b)$ and $(d))$ to the segments $A B$ and $C D$ for $R<I$ and $R>1$ respectively; if $R=1$ then the solutions are given by (3.10) and correspond to the critical point in the bisector of the first quadrant. These solutions amount to a monotonous branch in the bifurcation diagram, as sketched in figure 1(c), and all of them are globally, asymptotically stable as steady states of $(1.1),(1.2)$ (as analytically proven in [14] and numerically checked here for $-1<\alpha<1$ and $\alpha=1$ respectively).

(a.1) If $R<1$ then as $\lambda$ varies from $\lambda_{C}(>0)$ to $\infty$ the segment $A B$ moves from the origin upwards in figure $2(a)$. Thus. for moderate values of $\lambda-\lambda_{c}>0 . u_{s}$ and $v_{s}$ dominate in $-\frac{1}{2} \leqslant x<0$ and $0<x \leqslant \frac{1}{2}$ respectively, and the associated $x-t$ plot of the state variable $w$. defined by (2.4), (2.5) is a symmetric chevron, as that in figure $3(a)$. As $\lambda \rightarrow \infty$ the segment $A B$ approaches the heteroclinic orbits joining the critical points $(\lambda, 0)$, $(\lambda /(1+\alpha), \lambda /(1+\alpha))$ and $(0, \lambda)$, and thus the trajectory $A B$ spends the most part of the spatial domain $-\frac{1}{2}<x<\frac{1}{2}$ near the critical point $(\lambda /(1+\alpha), \lambda /(1+\alpha))$; the resulting $x-t$ diagram of the state variable $w$ shows a standing wave in the most part of the domain $-\frac{1}{2}<x<\frac{1}{2}$ (except near the end-walls $x= \pm \frac{1}{2}$ ), as in figure $3(b)$.

(a.2) If $R>1$ then as $\lambda$ varies from $\lambda_{C}(<0)$ to 0 the segment $C D$ moves in figure $2(d)$ from the origin upwards, and as $\lambda$ varies from 0 to $\infty$ the segment $C D$ moves downwards in figure 2(a). Thus, for moderate values of $\lambda-\lambda_{C}>0$ the $x-t$ diagram for the state variable $w$ shows a symmetric chevron, as in figure $3(c)$. As $\lambda \rightarrow \infty$. the segment $C D$ approaches the trajectories passing through the critical point $(\lambda /(1+\alpha), \lambda /(1+\alpha))$ and thus spend the most part of the spatial domain, $-\frac{1}{2}<x<\frac{1}{2}$, near the critical point, the associated $x-t$ diagrams for the state variable show a standing wave in the most part of the spatial domain, as in figure $3(d)$. The main difference with the case $R<1$ is that now the zones near the end-walls where the system exhibits travelling waves are much smaller and the values of the amplitudes of the wavetrains in these zones are much larger. Both differences are concerned with the extreme values of the reflection coefficient $\left(R=10^{-2}\right.$ and $\left.10^{2}\right)$ and the fact that as $R \rightarrow 0$ the ends of the segment $A B$ in figure $2,\left(u_{A}, v_{A}\right)$ and $\left(u_{B}, v_{B}\right)$. approach the critical points $(\lambda, 0)$ and $(0, \lambda)$. while as $R \rightarrow \infty$ the ends of the segment $C D$ are such that $\left(u_{C} / \lambda, v_{C} / \lambda\right) \rightarrow(\infty, 0)$ and $\left(u_{D} / \lambda, v_{D} / \lambda\right) \rightarrow(0, \infty)$ (and the points $(\infty, 0)$ and $(0, \infty)$ do not behave as critical points in the phase plane of (3.1)).

(a.3) If $R=1$ then the solution is given by (3.7) and the $x-t$ diagram for the state variable $w$ always shows a standing wave, as in the first plot in figure $5(c)$.

(b) If $\alpha>1$ then the symmetric steady states considered above are asymptotically stable and amount to a monotonous branch in $\lambda_{\mathrm{C}}<\lambda<\lambda_{\mathrm{SB}}$, where $\lambda_{\mathrm{SB}}$ will be calculated below. In addition, if $\lambda_{\mathrm{SB}}<\lambda<\lambda_{\mathrm{H}}$ (for some $\lambda_{\mathrm{H}}$ corresponding to a Hopf bifurcation point that will be considered below) then there are two non-symmetric, asvmptotically stable steady states that correspond in figure 2(c) to the segments $A^{\prime} B^{\prime}$ and $C^{\prime} D^{\prime}$ for $R<1$ and $R>1$ respectively, and to their symmetric ones under the reflection $u_{s} \leftrightarrow v_{s}$; if $R=1$ then these steady states correspond to the segments $E F$ and $F E$. In addition, if $\lambda$ is sufficiently large then there are other branches of symmetric and non-symmetric steady states that correspond in figure $2(c)$ to travelling along a closed orbit in a non-simply way; but they are unstable. The bifurcation diagram is always as sketched in figure $1(c)$. For illustration, 

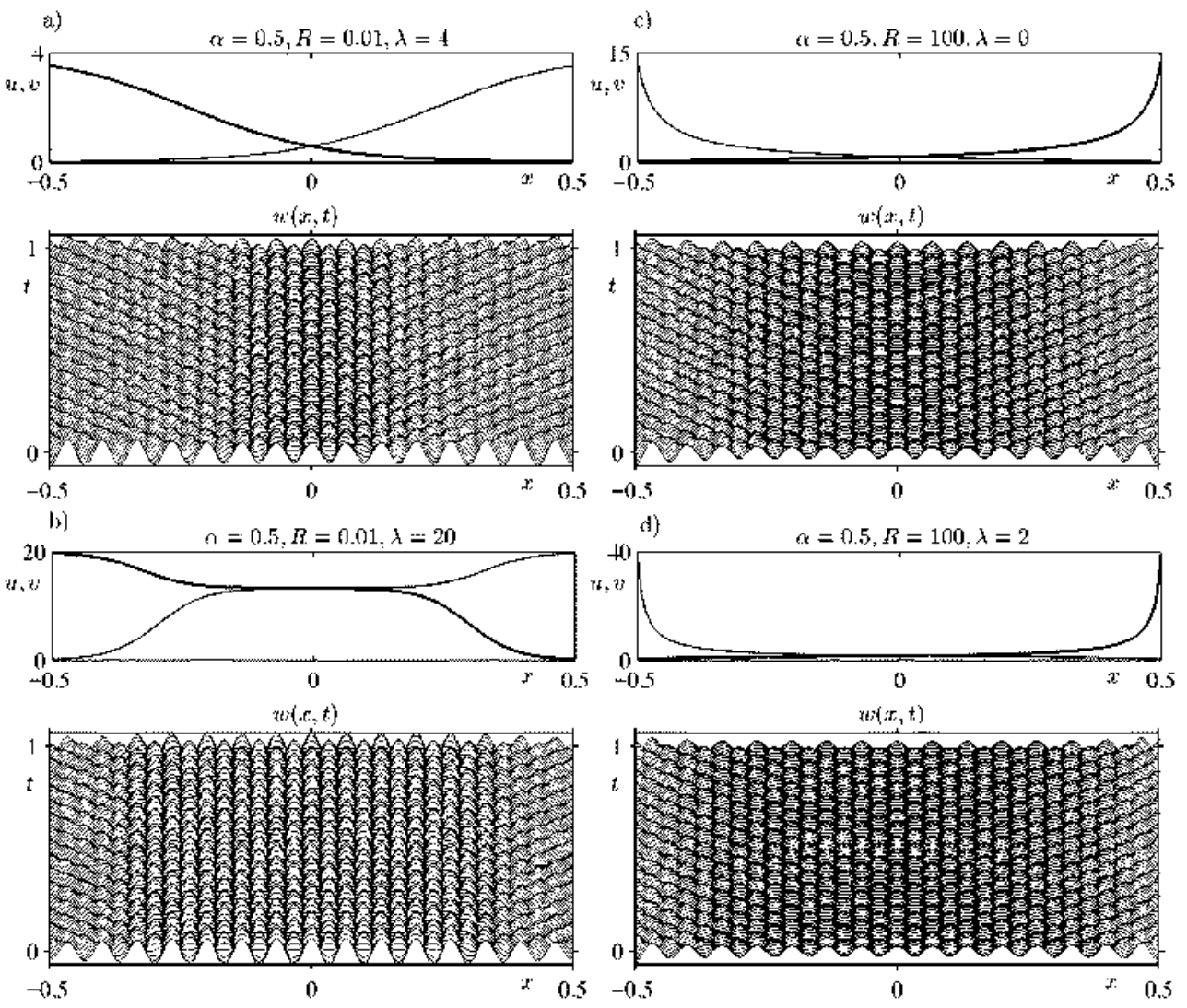

Figure 3. Spatial profiles of $u$ and $v$ and the corresponding $x-t$ diagrams of the state variable (2.4), (2.4) for representative values of the parameters $-1<\alpha<1, R$ and $\lambda$.

in figure 4 we give a part of the bifurcation diagrams for three representative pairs of values of the parameters $\alpha$ and $R$. For completion we have also plotted a part of the branch of periodic solution that bifurcates from the Hopf bifurcation point at $\lambda=\lambda_{\mathrm{H}}$. This branch will be completed in section 4 and has been calculated by a continuation method based on the discretization of the time-dependent problem (1.1), (1.2) described at the beginning of section 4 . The $x-t$ diagram of the state variable $w$ (defined by $(2.4) .\left(2.4^{\prime}\right)$ and (2.5)) is plotted in figure 5 for three representative points of each bifurcation diagram in figure 4. Note that symmetric and non-symmetric steady states correspond to symmetric and non-symmetric chevrons if $R \neq 1$ (figures $5(a)$ and $(b)$ ). and to standing waves and almost-pure trovelling waves if $R=1$ (figure 5(c)): limit cycles are non-symmetric. and correspond to beating states (around the formerly stable non-symmetric steady solution) in the three cases. Again, there are qualitative differences between the steady states in the cases $R<1$ and $R>1$, which may be explained in terms of the phase portraits of figure 2 as follows. The points in the segments $A^{\prime} B^{\prime}$ and $C^{\prime} D^{\prime}$ where $u_{s}=v_{s}$ approach the critical point $\left(u_{s}, v_{s}\right)=(0,0)$ if $R<1$ and the ordinary point $\left(u_{s}, v_{s}\right)=(\lambda / 2, \lambda / 2)$ if $R>1$. Consequently that part of the spatial domain where both counter-propagating wavetrains coexist (with similar activity) is much larger if $R<I$ than if $R>I$. and the values of the amplitudes of the wavetrains there are much smaller if $R<I$ than if $R>I$ (compare 

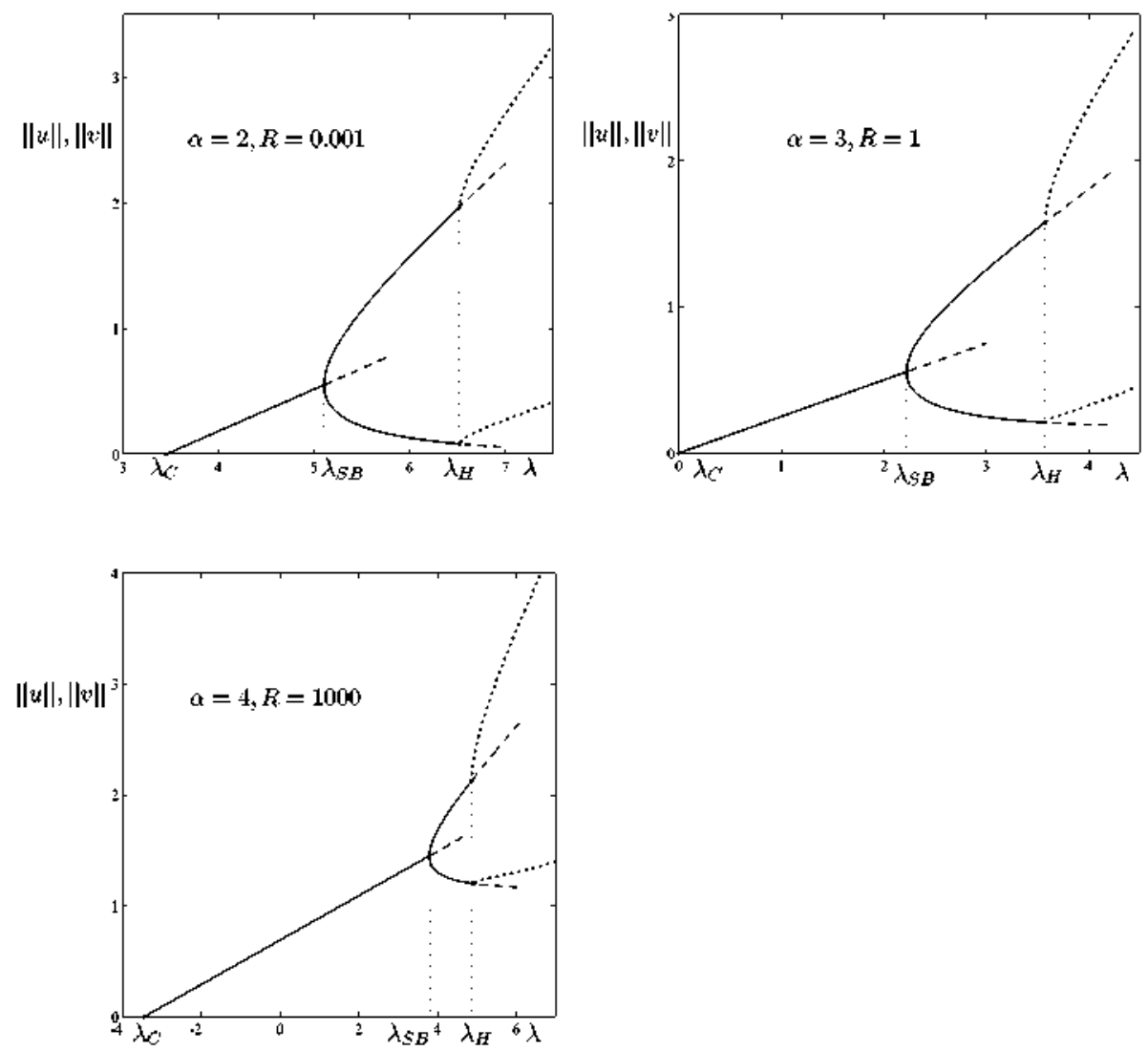

Figure 4. Bifurcation diagrans of the solutions of (1.1)-(1.3) for three representative sets of values of the paraneters $\alpha>1$ and $R$.

figures $5(a)$ and $(b))$. In addition, since the periodic solutions are oscillations around the steady states, there are also qualitative differences between the periodic solutions. The non-steady nature of these solutions is appreciated mainly in that part of the spatial domain where the amplitudes of the wavetrains are smallest: namely. it is appreciated in the timedependence of (the size and position of the region where both counter-propagating waves dominate if $R<I$ (as in figure $5(a)$ ), and in the quantitative time dependence of both amplitudes near the end-wall where they are smallest if $R>1$ (as in figure $5(b)$ ).

Let us now consider the bifurcation values $\lambda_{\mathrm{SB}}$ and $\lambda_{\mathrm{H}}$. associated with symmetrybreaking and Hopf bifurcation.

If $R=1$ then symmetry breaking takes place when the constant solutions (3.10) lose stability; since the coefficients in (3.4) are constant in this case, the linear problem (3.4), (3.5) is readily solved in closed-form to obtain

$$
\lambda_{\mathrm{SB}}=(\pi / 2)[(\alpha+1) /(\alpha-1)]^{1 / 2} .
$$

If $R \neq 1$ then symmetry breaking takes place (when symmetric and non-symmetric solutions coalesce, that is) precisely when symmetric solutions are such that the associated segment ( $A B$ or $C D$, depending on whether $R<1$ or $R>1$ ) in figure $2(c)$ is tangent to the straight line $u_{s}=R v_{s}$. When using this tangency condition, the first integral (3.11) and equations (3.1) and (3.2). the value of the bifurcation parameter at symmetry breaking is 

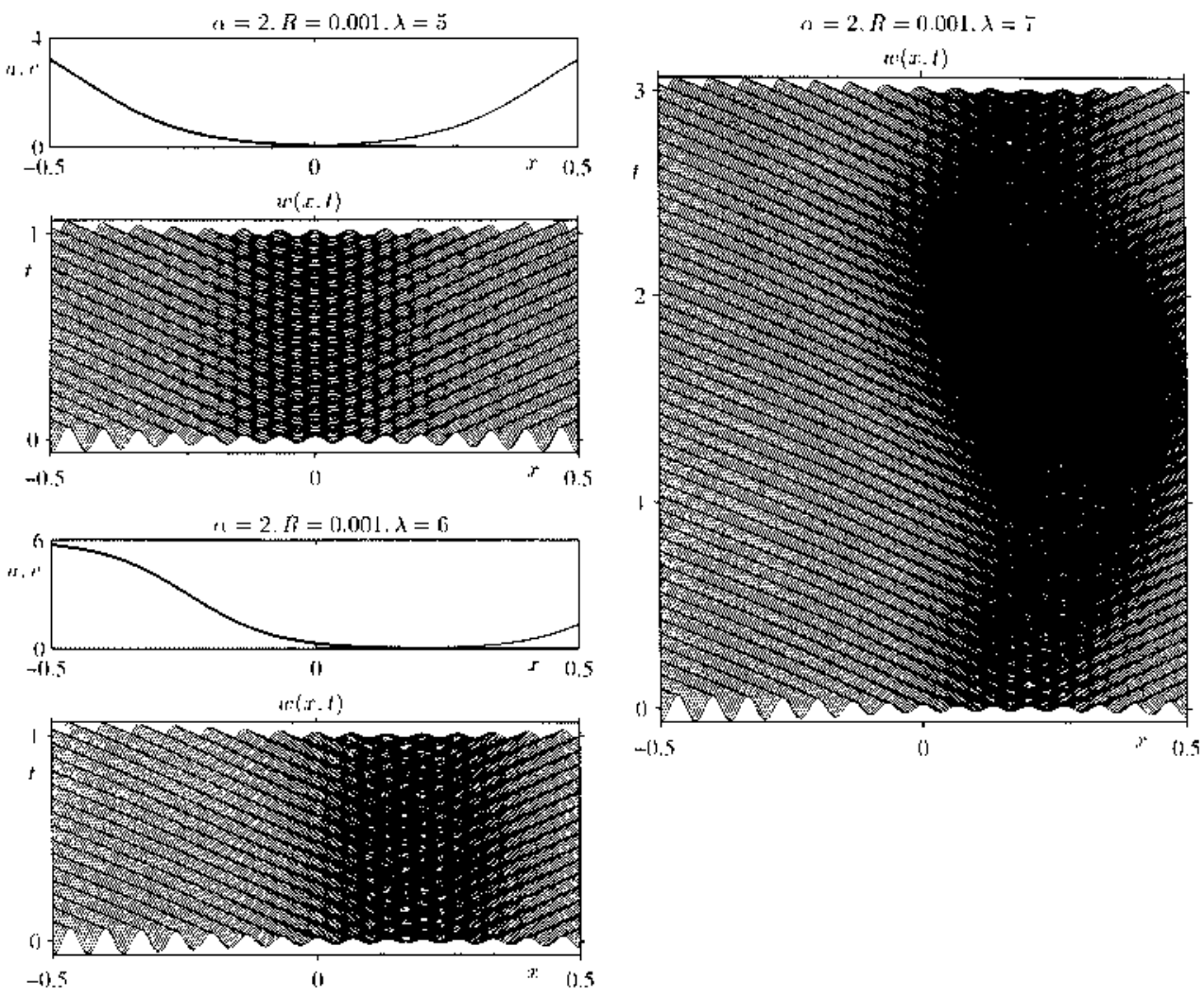

(a)

Figure 5. Spatial profiles and $x-t$ diagrans for a symmetric and non-symmetric steady solution and $x-t$ diagram corresponding to a periodic solution, for: $(a) \alpha=2, R=0.001$, (b) $\alpha=4, R=1000$ and (c) $\alpha=3, R=1$.

found to be as given by

$$
\lambda_{\mathrm{SB}}=\left|\int_{z_{0}}^{z_{1}}(1-z)^{-1} F(z)^{-1 / 2} \mathrm{~d} z\right|
$$

where

$$
\begin{aligned}
& F(z)=z^{2}-\delta|1-z|^{1-\alpha} \\
& \delta=4 R(\alpha-1)^{\alpha-1} /\left[(R+1)^{2}(\alpha+1)^{\alpha+1}\right] \\
& z_{0}=2 /(\alpha+1)
\end{aligned}
$$

while $z_{1}$ is defined as follows. If $R<1$ then $z_{1}$ is the smallest positive root of $F(z)=0$ and if $R>1$ then $z_{1}$ is the largest root of $F(z)=0$ such that $z_{1}<1$; note that $\lambda_{\mathrm{SB}}$ is always positive. Also, as $R \rightarrow 1 \lambda_{\mathrm{SB}}$ is seen to converge to that value given in (3.12), that was obtained by a somewhat different definition of symmetry breaking. For convenience we also give the following asymptotic expressions, that are obtained from (3.12),

$$
\begin{aligned}
& \lambda_{\mathrm{SB}}=[-\log R+(\alpha+1) \log ((\alpha+1) /(\alpha-1))+o(1)] / 2 \quad \text { as } R \rightarrow 0^{+} \\
& \lambda_{\mathrm{SB}}=[\log R+(\alpha+1) \log ((\alpha+1) / 2)+o(1)] /(\alpha-1) \quad \text { as } R \rightarrow \infty .
\end{aligned}
$$



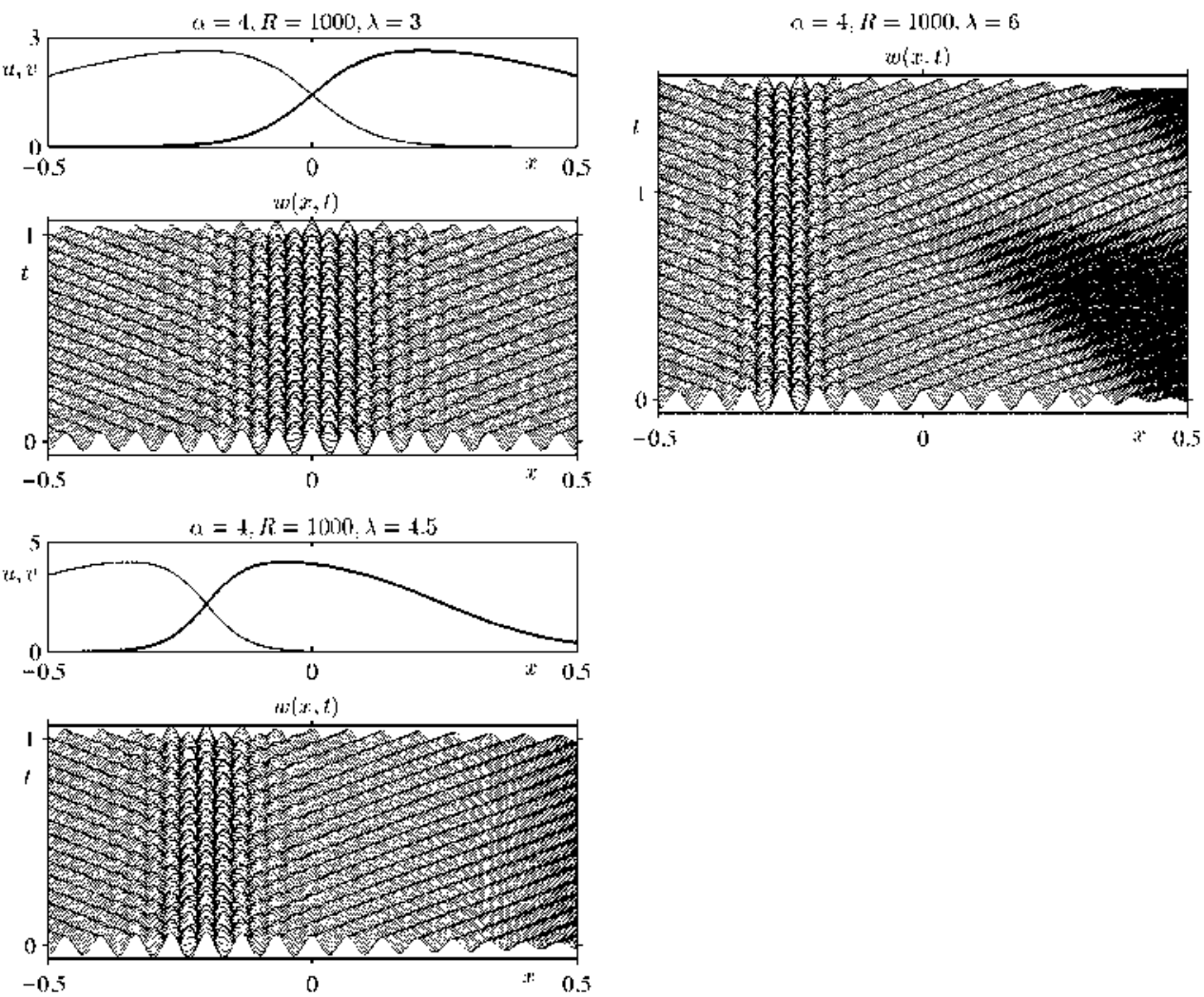

(b)

Figure 5. (Continued)

The Hopf bifurcation point is calculated as the upper instability limit of the branch of non-symmetric solutions. The associated value of the bifurcation parameter, $\lambda_{\mathrm{H}}$, is numerically calculated in terms of $R$ and $\alpha$ by means of a continuation method [16] applied to the equation $\operatorname{Re} \omega=0$, where $\operatorname{Re}$ stands for the real part, $\omega$ is an eigenvalue of (3.4), (3.5) and $\left(u_{s}, v_{s}\right)$ is a non-symmetric solution of (3.1), (3.2). At each step in the continuation process the problems (3.1), (3.2) and (3.4), (3.5) are solved by a multiparametric slooting method. In order to initiate the process. the problem (1.4). (1.5) is discretized by a finitedifferences scheme to obtain sufficiently good approximations of the eigenvalue whose real part is largest (this discretization is also used to analyse the stability of the steady states of (1.1), (1.2)); then the parameter $\lambda$ is varied (for fixed values of $R$ and $\alpha$ ) until the real part of the eigenvalue is conveniently small.

Now, the three bifurcation values considered above, $\lambda_{\mathrm{C}}, \lambda_{\mathrm{SB}}$ and $\lambda_{\mathrm{H}}$, are plotted versus $R$, for representative values of $\alpha$, in figure 6 ; for convenience, the (limiting value of the) period $T$ of the bifurcated solutions at the Hopf bifurcation point is also given. Note that $0<\lambda_{\mathrm{SB}}<\lambda_{\mathrm{H}}$ as indicated above, and that both $\lambda_{\mathrm{SB}} / \log R$ and $\lambda_{\mathrm{H}} / \log R$ converge to a constant as $R \rightarrow 0$ and $R \rightarrow \infty$ (this behaviour was to be expected for $\lambda_{\mathrm{SB}}$, see (3.13), (3.14))

Finally, let us point out that if the end-walls were amplifying then $u$ and $v$ should be interchanged in (2.4) and $\left(2.4^{\prime}\right)$ ). and some of the $x-t$ diagrams in figures 3 and 5 (i.e. 

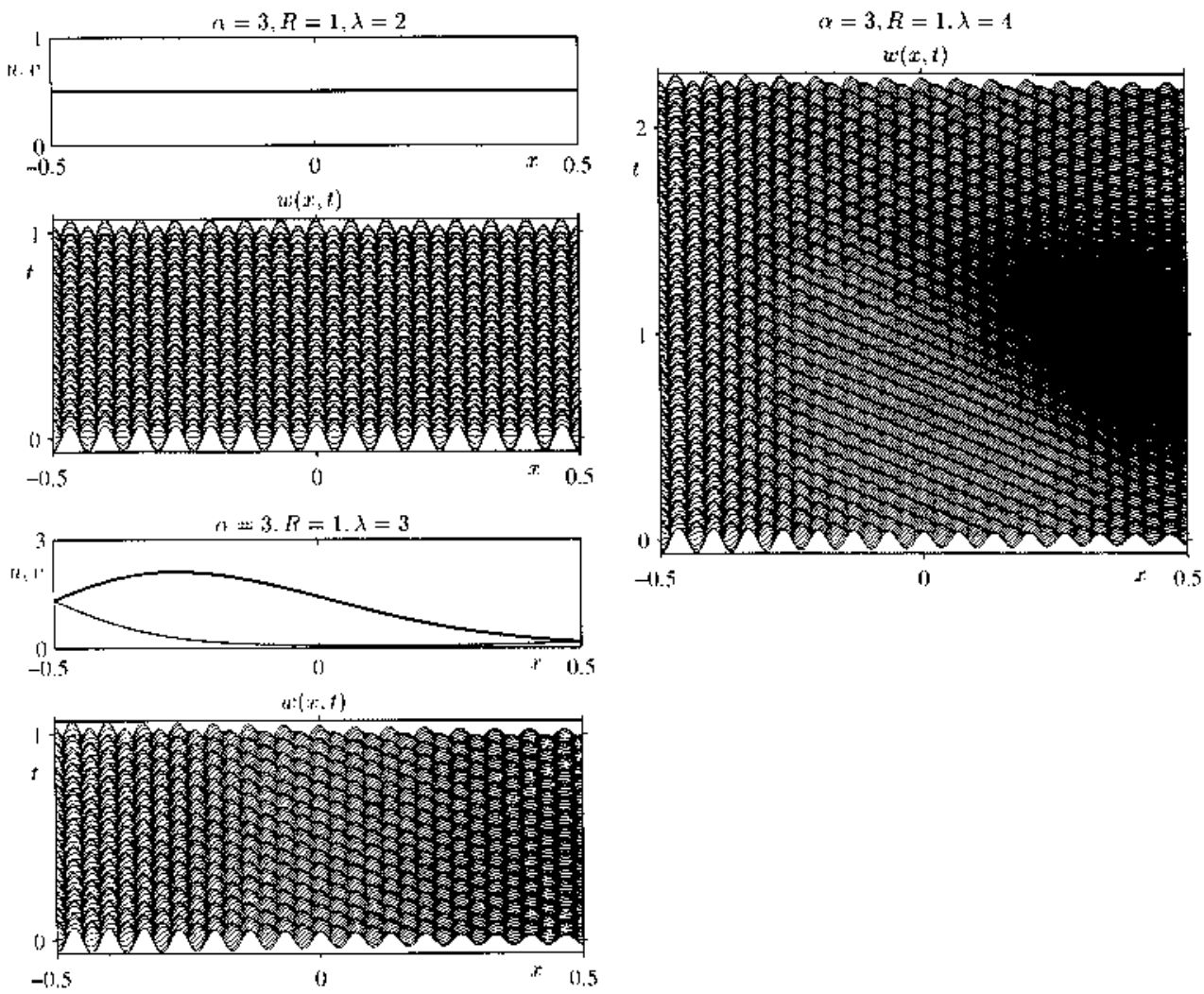

(c)

Figure 5. (Continued)

those corresponding to steady states of (1.1). (1.2)) would essentially stand after reversing the time variable.

\section{More complex dynamics $(\alpha>1)$}

Let us now analyse the dynamics of (1.1), (1.2) for larger values of the bifurcation parameter $\lambda$ than those considered in section 3 . This will be done as follows. The problem (1.1)-(1.3) will be numerically integrated by using a standard explicit, second-order, predictor-corrector method along the characteristics, with $\Delta t=\Delta x / 2$. Then we shall calculate the sections of the orbits, for a sufficiently large time, on the hyperplane $H$ defined as

$$
\|u\|_{L_{1}}+\|v\|_{L_{1}} \equiv \int_{-1 / 2}^{1 / 2}(u+v) \mathrm{d} x=(2 \lambda+\log R) /(\mathbf{I}+\alpha) .
$$

This hyperplane has been chosen because the periodic orbits of (1.1), (1.2) intersect $H$ in each period. In order to prove this assertion, divide the first and second equations in (1.1) by $u$ and $v$ respectively, integrate in $-\frac{1}{2}<x<\frac{1}{2}$, add and take into account the boundary conditions (1.2) to obtain

$$
\frac{1}{2} \frac{\mathrm{d}}{\mathrm{d} t} \int_{-1 / 2}^{1 / 2} \log (u v) \mathrm{d} x=2 \lambda+\log R-(1+\alpha) \int_{-1 / 2}^{1 / 2}(u+v) \mathrm{d} x .
$$



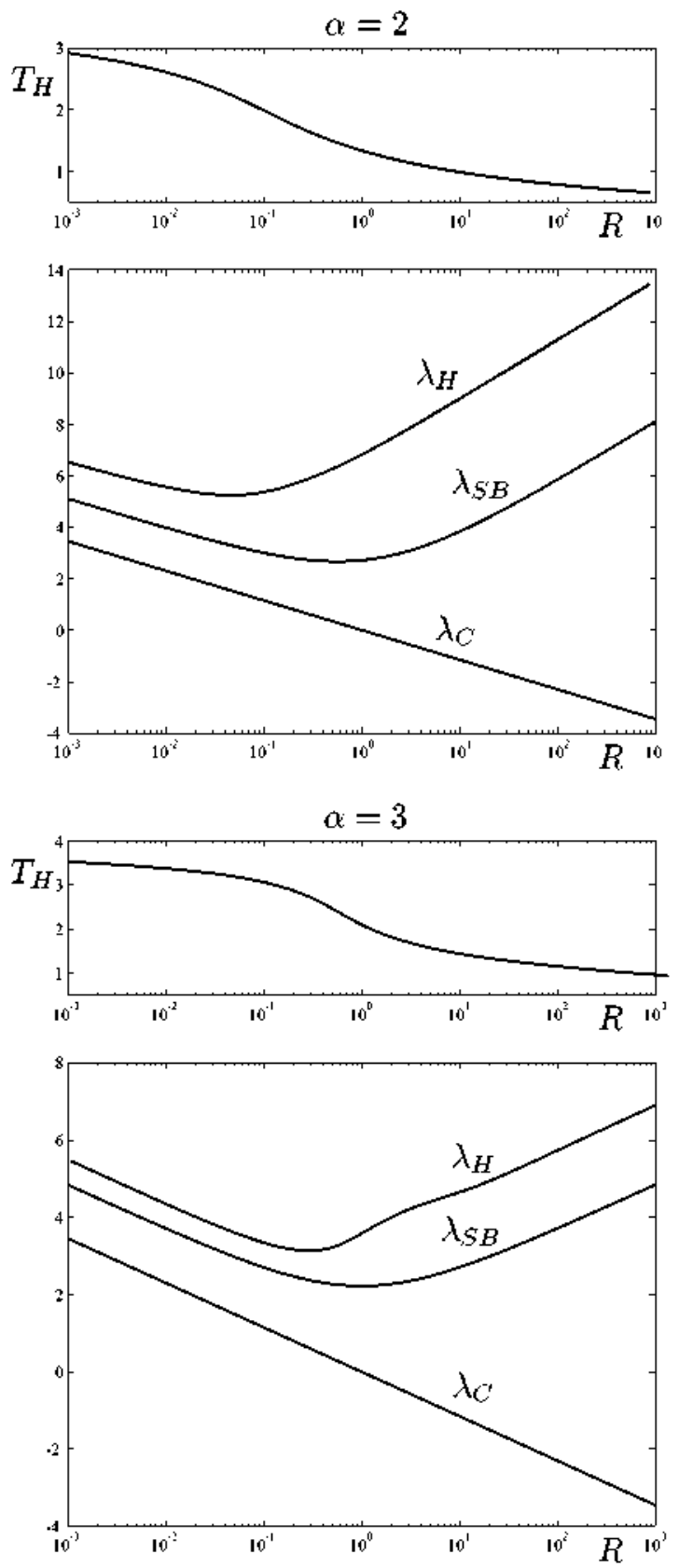

Figure 6. Bifurcation values $\lambda_{\mathrm{C}}, \lambda_{\mathrm{SB}}$ and $\lambda_{\mathrm{H}}$ versus $R$ for three different values of $\alpha$. Upper plots show the period of the solution, $T_{\mathrm{H}}$, at the Hopf bifurcation.

Note that the right-hand side of this equation vanishes precisely on $H$. Assume for contradiction that a periodic orbit does not intersect $H$. Then the right-hand side of (4.2) has a constant sign and the function $t \rightarrow \int_{-1 / 2}^{1 / 2} \log (w v) \mathrm{d} x$ is strictly monotonous. But this cannot happen because this function is periodic. 

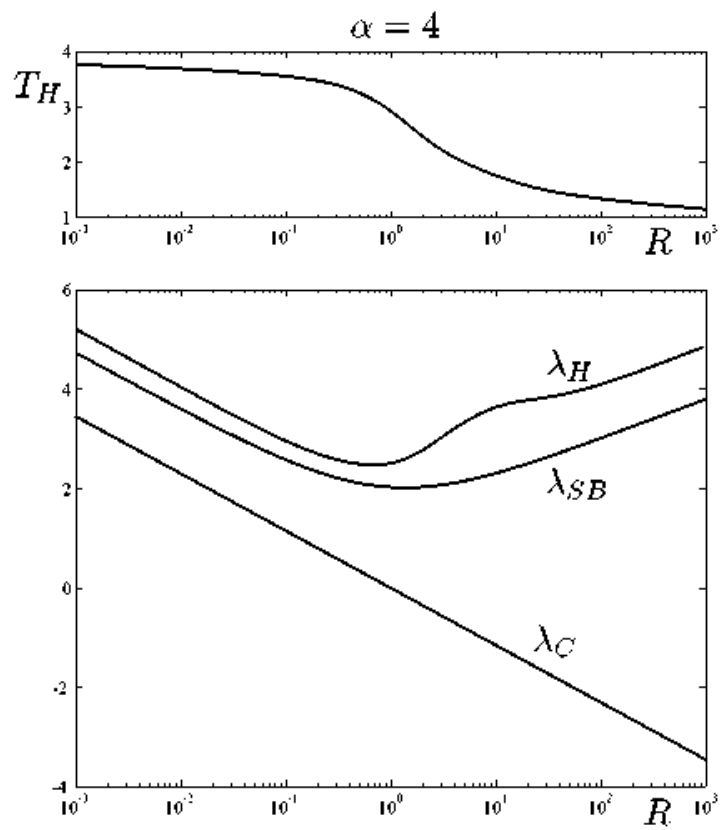

Figure 6. (Continted)

Now the problem (1.1), (1.2) is invariant under the symmetry

$$
x \rightarrow-x \quad u \leftrightarrow v
$$

that results from the spatial reflection symmetry of the underlying physical problem. which was mentioned right after equations (1.6) and (1.7). Then this symmetry has a plysical interest. It is also relevant from the mathematical point of view because several transitions in the bifurcation diagrams below will be associated with symmetry breaking and symmetricgaining processes. In order to appreciate this symmetry we shall use the following coordinate on the hyperplane (4.1) to plot the attractors (see figure 7)

$$
d_{p}=\left(\|v\|_{L_{1}}-\|u\|_{L_{1}}\right) / \sqrt{2}=\int_{-1 / 2}^{1 / 2}(v-u) / \sqrt{2} \mathrm{~d} x .
$$

Note that when plotting the intersections of an orbit on the hyperplane with this coordinate, the resulting set of points is symmetric on the origin if and only if the orbit is invariant under the symmetry (4.3).

Periodic orbits exhibit a finite number of intersections on the lyperplane. and aperiodic orbits associated with chaotic attractors exhibit infinitely many intersections, which accumulate on a Cantor-set-like structure. In order to plot chaotic attractors. an appropriately large (but of course finite) number of intersections will be plotted (for the structure of the attractor to be somehow appreciated), after integrating (1.1), (1.2) in an appropriately large initial interval, to eliminate the initial transient when the orbit approaches the attractor. In order to ascertain chaotic dynamics, the first few Lyapunov exponents will be numerically calculated as usual [17], by integrating (1.1), (1.2) once and integrating the associated linearized problem for several, linearly independent initial conditions, and applying a GranSchmidt re-orthonormalization procedure at $t=T, 2 T \ldots$ for some fixed $T$. Finally. some unstable branches of periodic solutions will also be needed to complete some parts of the 


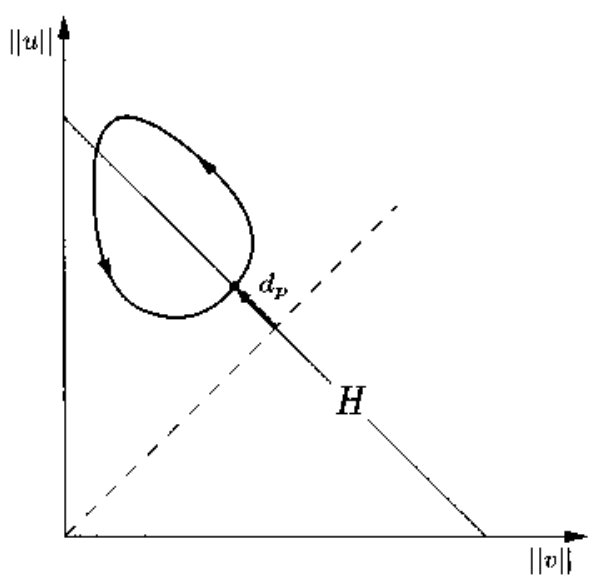

Figure 7. Intersection of a periodic orbit with the hyperplane $H$ showing the coordinate used, $d_{P}$.

bifurcation diagrams. These branches are calculated by means of a numerical continuation method [16].

Now, for larger values of the bifurcation parameter than those considered in section 3 , the problem (1.1), (1.2) exhibits a fairly large variety of transitions whose details depend on $R$ and $\alpha$. We do not intend on classifying the bifurcation diagrams, as we did in section 2 . Instead we shall only consider three cases, namely, those considered in figure 4 .

The bifurcation diagram for $\alpha=2$ and $R=0.001$ is given in figure 8 where all attractors are non-symmetric and the following main features are observed. If $\lambda_{\mathrm{H}}=6.53<\lambda<\lambda_{1} \simeq$ 8.38 then there is a branch of non-symmetric stable periodic solutions (i.e. the continuation of that plotted in figure 4) that exhibits a flip (period-doubling) bifurcation at $\lambda=\lambda_{1}$. For larger values of $\lambda$ there is a period-doubling sequence, at $\lambda=9.52, \ldots$, that accumulates at $\lambda=\lambda_{2} \simeq 9.64$. If $\lambda_{2}<\lambda<\lambda_{3} \simeq 11.17$ there is a chaotic attractor that alternates with periodic windows. as is usually the case after a period-doubling sequence. At $\lambda=\lambda_{3}$ the chaotic attractor collides with an unstable branch of non-symmetric periodic solutions and disappears. A second branch of stable, periodic solutions exists for $\lambda_{4} \leqslant \lambda<\lambda_{5}$, where $\lambda_{4} \simeq 11.11$ and $\lambda_{5} \simeq 11.69$, that exhibits a new period doubling sequence that accumulates at $\lambda=\lambda_{6} \simeq 12.18$. Note that if $\lambda_{4} \leqslant \lambda \leqslant \lambda_{3}$ then there is a hysteresis between the branch of periodic solutions and the chaotic attractor. For $\lambda>\lambda_{6}$ a new chaotic attractor appears that (again alternates with periodic windows and) collides with the unstable branch of periodic solutions at $\lambda=\lambda_{7} \simeq 12.63$, and enlarges. Note that the new branch that appears (in the upper part of the plot in figure 8 ) at $\lambda \simeq 8.9$ is just due to an additional intersection of the attractor described above with the hyperplane $H$. and not to new dynamical features (i.e. period doublings or qualitative enlargement of the attractor). The spatio-temporal picture of the (periodic or chaotic) attractors plotted in figure 8 is always qualitatively similar to the third picture in figure $5(a)$; the main difference is that the behaviour is now more complex. As an example, the $x-t$ diagram of the periodic (period $\simeq 3.06$ ) attractor at $\lambda=11.5$ is given in figure 9.

The bifurcation diagram for $\alpha=3$ and $R=1$ is given in figure 10. If $\lambda_{\mathrm{H}} \simeq 3.58<$ $\lambda<\lambda_{1} \simeq 5.46$ then there is a branch of non-symmetric, stable, periodic solutions (i.e. the continuation of that plotted in figure 4 ) that loses stability through a subcritical perioddoubling bifurcation at $\lambda=\lambda_{1}$ (a part of the branch of unstable period-doubled solutions is plotted as a broken curve in figure 10 ). For $\lambda>\lambda_{1}$ the system jumps to a new branch 


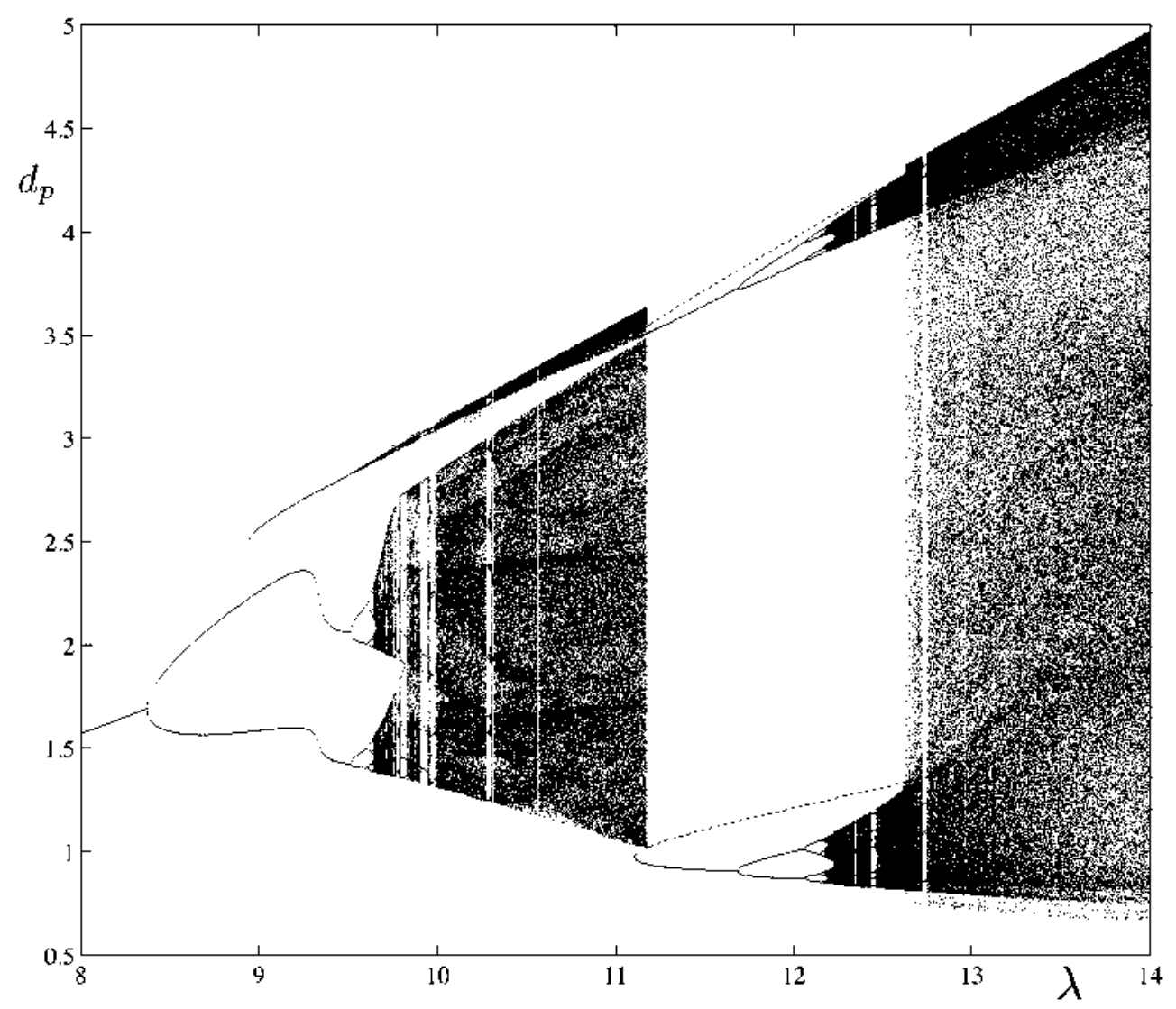

Figure 8. Poincare sections of the solutions of $(1.1)-(1.3)$ for $\alpha=2$ and $R=0.001$.

of stable, symmetric solutions. with a period about four times the period of the formerly stable non-symmetric solutions. If $\lambda$ is decreased from $\lambda_{1}$ then the new symmetric branch exhibits a symmetry-breaking bifurcation at $\lambda=\lambda_{2} \simeq 5.42\left(<\lambda_{1}\right)$, and the bifurcated non-symmetric solutions become unstable at $\lambda=\lambda_{3} \simeq 5.41\left(<\lambda_{2}\right)$ through a saddle-node bifurcation. Note that there is a hysteresis between the above-mentioned stable branches in the range $\lambda_{3}<\lambda<\lambda_{1}$. For larger values of $\lambda$ the symmetric branch loses symmetry at $\lambda=\lambda_{4} \simeq 9.12$ and subsequently exhibits a first period-doubling at $\lambda=\lambda_{5} \simeq 9.62$ and then a period-doubling sequence that accumulates at $\lambda=\lambda_{6} \simeq 9.77$. The resulting chaotic attractor (that alternates with periodic windows as usual) gains symmetry at $\lambda=\lambda_{7} \simeq 9.89$. The spatio-temporal picture of the non-symmetric solutions in $\lambda_{\mathrm{H}}<\lambda<\lambda_{1}$ is always qualitatively similar to that in third plot in figure $5(\mathrm{c})$. The attractor in $\lambda_{3}<\lambda<\lambda_{7}$ exhibits a spatio-temporal picture that is always (albeit of whether it is symmetric or nonsymmetric, periodic or chaotic) similar to that plotted in figure 11(a), corresponding to $\lambda=7.4$. For $\lambda_{7}<\lambda \leqslant 12$ the attractor exhibits an intermittent behaviour that is similar to that in figure $11(a)$, except for some sudden excursions to a qualitatively different dynamics: the $x-t$ diagram of one such excursion for $\lambda=11$ is plotted in figure 11 $(b)$. Again, this chaotic behaviour alternates with periodic windows

Finally, the bifurcation diagram for $\alpha=4$ and $R=1000$ is plotted in figure 12 . where again the lower branch of non-symmetric. stable, periodic solutions in $7 \leqslant \lambda<\lambda_{1} \simeq 8.44$ 


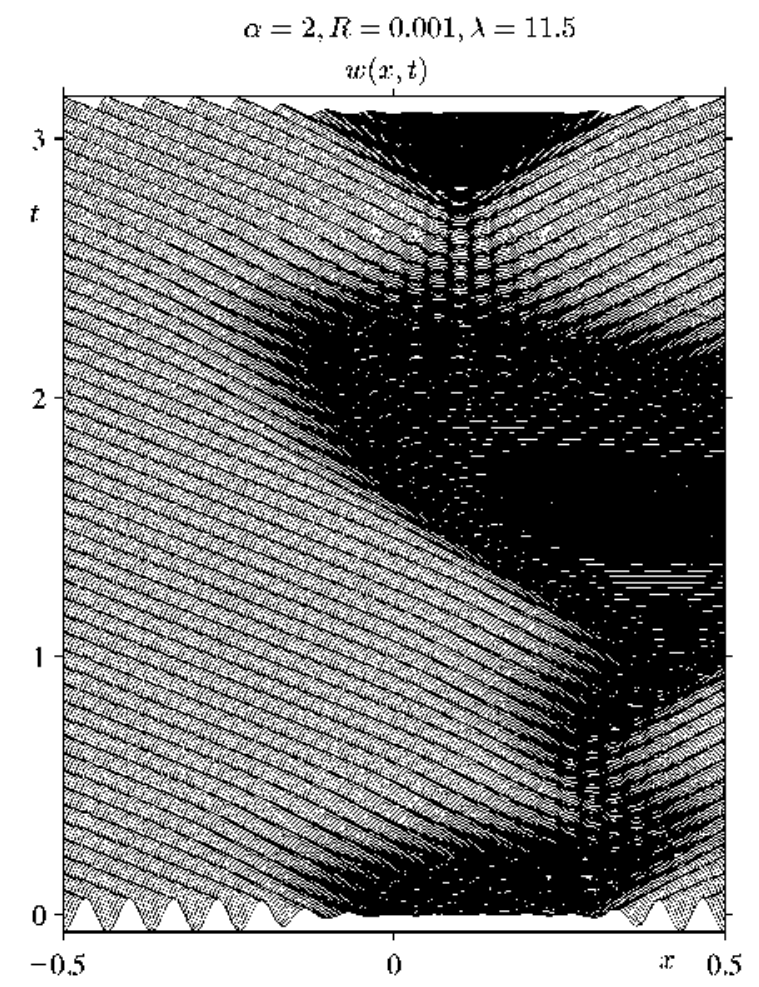

Figure 9. $x-t$ diagran for $\lambda=11.5$ in figure 8 .

is the continuation of that plotted in figure 4 . That branch exhibits a saddle-node bifurcation at $\lambda=\lambda_{1}$ and the resulting (reversed) branch of unstable solutions bends again at $\lambda=\lambda_{2} \simeq 7.24<\lambda_{1}$, and becomes stable. That stable branch loses stability at $\lambda=\lambda_{3} \simeq 7.42$, where an invariant, stable. non-symmetric torus bifurcates supercritically. In $\lambda_{3}<\lambda<\lambda_{4} \simeq 10.74$ the torus exhibits several symmetry-gaining and symmetry-breaking bifurcations (see figure 12) and the associated solutions appear as quasiperiodic except in some periodic windows, some of them (e.g. that in $10.4 \leqslant \lambda \leqslant 10.6$ ) exhibiting fairly large periods. Note that in $\lambda_{2}<\lambda<\lambda_{1}$ the system exhibits hysteresis, between a periodic attractor and either a periodic or a quasiperiodic attractor. The torus exhibits a qualitative change at $\lambda=\lambda_{4}$; the new (periodic or quasiperiodic) behaviour in $\lambda_{4}<\lambda<\lambda_{5} \simeq 10.92$ corresponds qualitatively to oscillations around the clearly non-symmetric solutions that disappeared at $\lambda=\lambda_{1}$ and their symmetric ones. while the former (periodic or quasiperiodic) behaviour in $\lambda_{3}<\lambda<\lambda_{4}$ may be qualitatively seen as an oscillation around the more symmetric periodic solutions that existed and were stable in $\lambda_{2}<\lambda<\lambda_{3}$. The torus breaks down at $\lambda=\lambda_{5}$ where a new, more complex attractor appears, the associated solutions being chaotic, with two positive Lyapunov exponents, except in some periodic windows. The spatio-temporal picture of the periodic solutions in the lower branch, in $7<\lambda<\lambda_{1}$, are qualitatively similar to that in the third plot in figure $5(b)$, while the $x-t$ diagrams of other, qualitatively different, representative solutions are given in figure 13 . Note that the periodic solution in figure 13 $(a)$ (corresponding to the upper periodic branch at $\lambda=7.25$ ) is approximately symmetric. Also the quasiperiodic solution in figure $13(b)$ is symmetric, and may be seen as the result of a symmetry-gaining process from the solution in figure $13(a)$. 


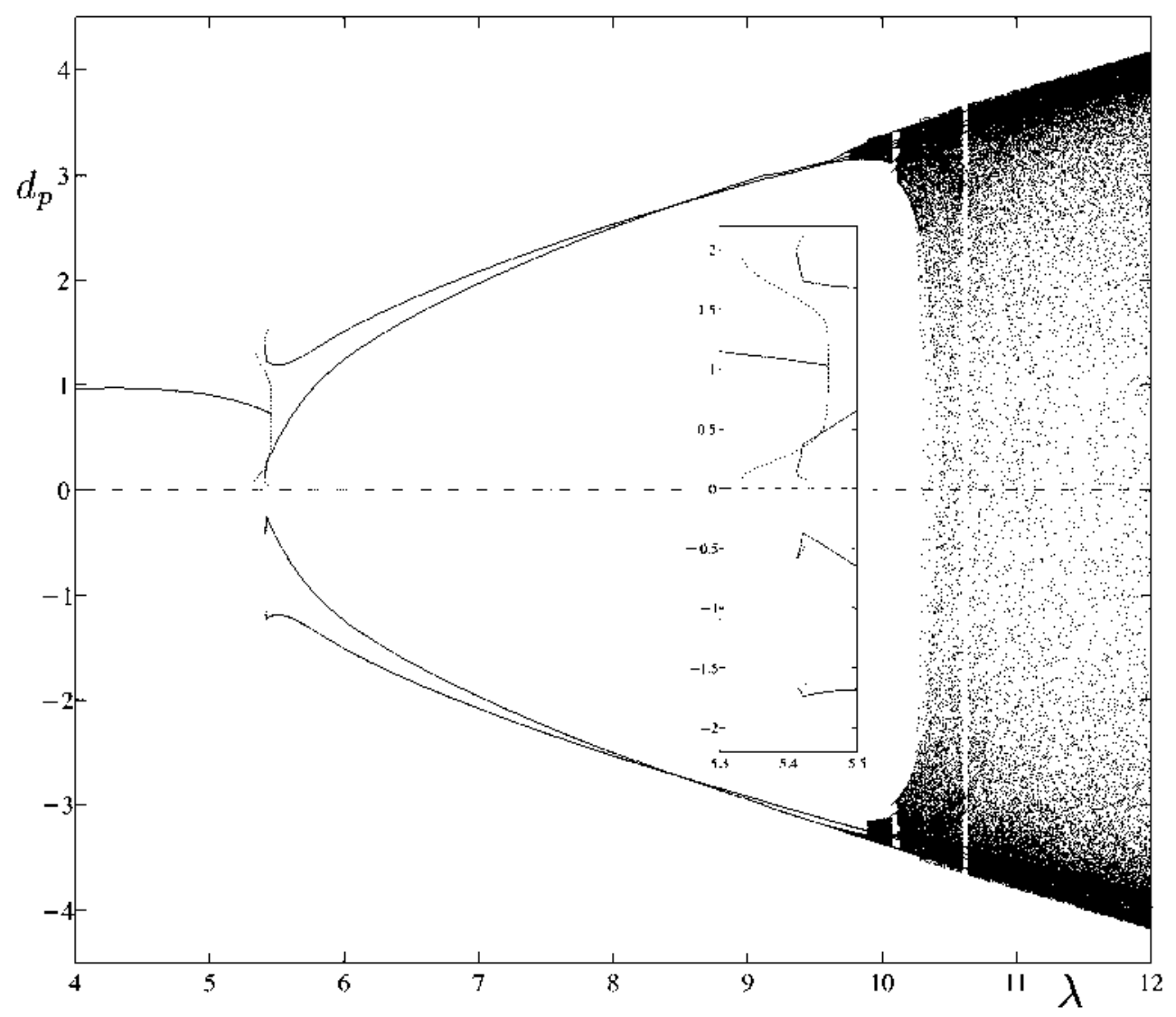

Figure 10. Poincare sections of the solutions of (1.1)-(1.3) for $\alpha=3$ and $R=1$ (inset: enlargement of the region near $\lambda=5.4$ ).

The chaotic solution in figure $13(c)$ may be qualitatively seen as an alternating state, between the non-symmetric periodic solution in figure $5(b)$ and its symmetric one.

A remark about the numerical results described above for $\alpha>1$ is now in order. As indicated above, the first three bifurcations in figure 4 are always present. For $\lambda>\lambda_{\mathrm{H}}$ instead, the qualitative shape of the bifurcation diagram largely depends on the specific values of the parameters $R$ and $\alpha$. Thus, we do not claim that the results in figures 8,10 and 12 are representative of those for other values of the parameters; they are just examples of the complex behaviour of the solutions of $(1.1),(1.2)$ as $\lambda$ increases. It is fairly costly to obtain bifurcation diagrams and thus we have been unable to obtain sufficiently many of them to get precise general conclusions. Nevertheless. some additional numerics not presented here allow us to draw two conclusions. First, chaotic dynamics is the rule (and not the exception) as $\lambda$ increases, and the mechanisms for the appearance of chaos are the usual ones. Namely, period-doubling sequences, intermittency and bifurcation to tori, the first two of them being the most frequent ones if $R<1$ (i.e. if the group velocity is positive for absorbing boundaries, as indicated above), while quasiperiodic solutions seem to appear most frequently if $R>1$ (negative group velocities). A second conclusion is that the symmetry (4.3) plays an essential role in the bifurcation diagrams. which frequently (but not always. see figure 8) exhibit several symmetry-gaining and symmetry-breaking bifurcations. 
at)

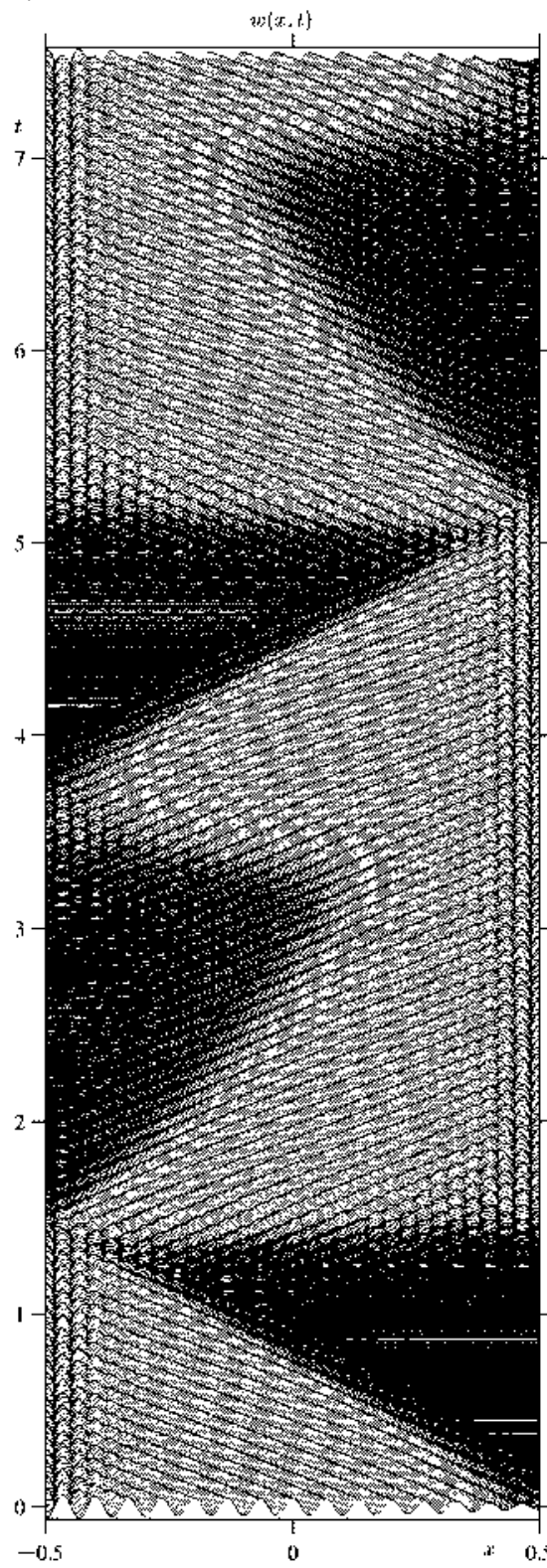

b) $\quad a=3, R=1, \lambda=1$

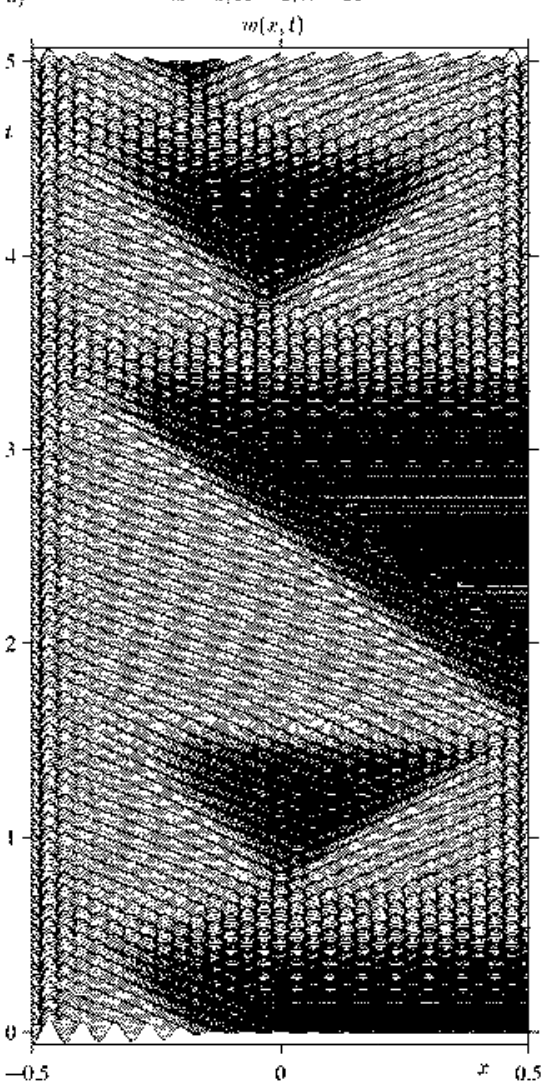

Figure 11. $x-t$ diagrams for $\lambda=8$ and 11 in figure 10 .

Finally, note that the complex dynamics described above is entirely due to the interplay of the coupling nonlinear effects (i.e. the terms $-2 \alpha u w$ in equations (1.1)) and the interaction with the end-walls (i.e. the reflection of the wavetrains that leads to the linear boundary conditions (1.2)). This is so because a third complexity-leading mechanism (associated with the intermediate scales that are considered next), resulting from dispersion. is absent in the hyperbolic model (1.1)-(1.3). 


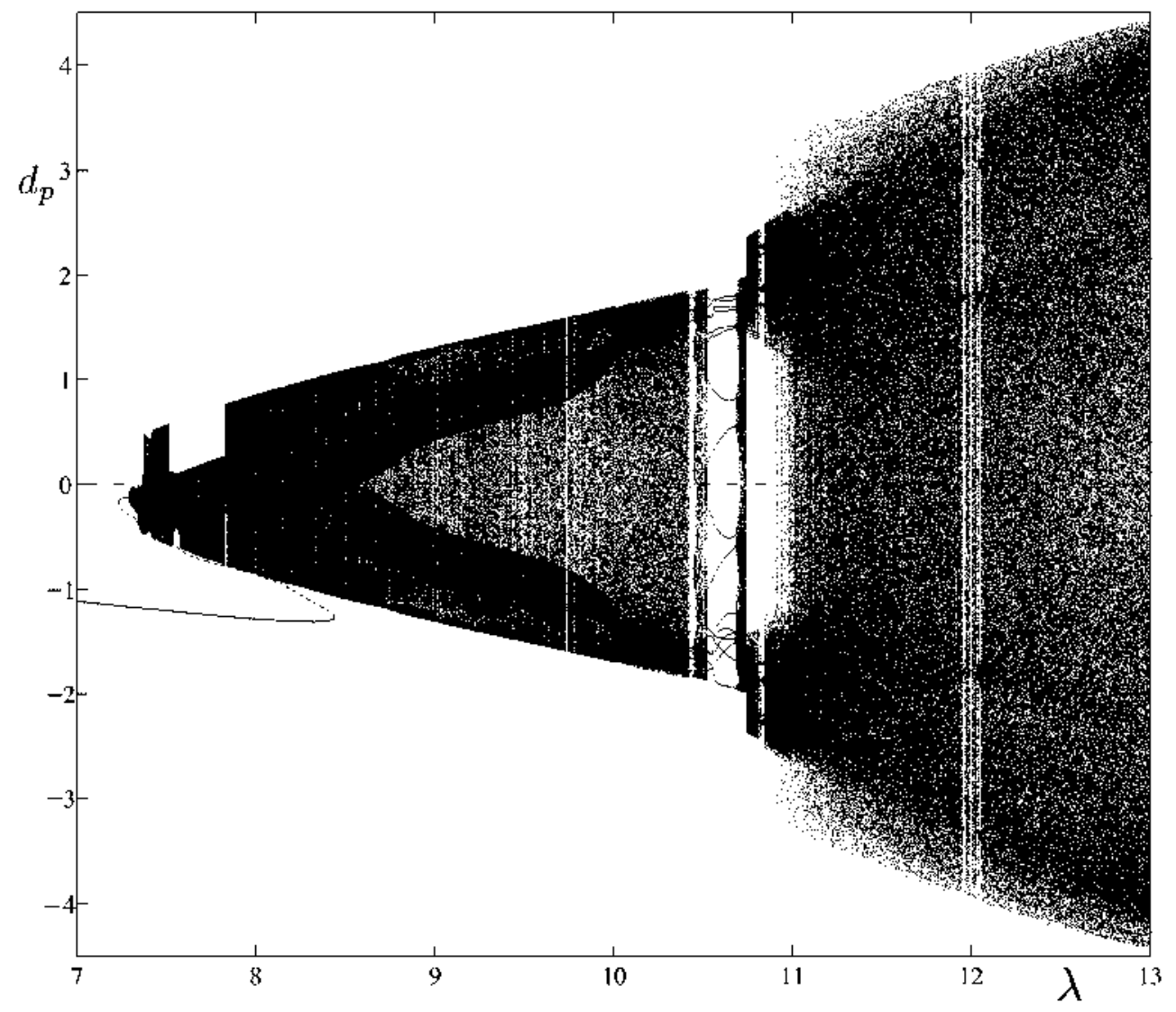

Figure 12. Poincare sections of the solutions of (1.1)-(1.3) for $\alpha=4$ and $R=1000$.

\section{The intermediate scales}

As pointed out in section 2, the hyperbolic system (1.1), (1.2) is derived from the amplitude equations (1.19)-(1.23) under the assumption that the terms containing second spatial derivatives, which account for diffusion and dispersion, may be neglected. In this case the complex amplitudes $A$ and $B$ never vanish (provided that they do not vanish initially) according to property (i) at the end of section 2 , and the number of wavelengths associated with both wavetrains is conserved. In addition, the phases of $A$ and $B$ behave quite regularly in the timescale associated with (1.1). (1.2). according to remark (b), right after equation (2.2). The situation is completely different when dispersion and diffusion come into play through intermediate scales, whose size is in between the basic wavelength of the wavetrains and the size of the domain. Then both the amplitudes and phases of $A$ and $B$ may exhibit complex spatio-temporal behaviour, and the number of wavelengths can change as time proceeds yielding dislocations in spacetime diagrams.

The incipient evolution of intermediate scales will be analysed in section 5.1, where we shall derive a linear equation giving the evolution of a generic (small) perturbation containing intermediate scales (added to a solution without these scales). That equation will allow us to inquire into whether the intermediate scales are exponentially damped out as time proceeds or whether they grow exponentially: to this end we shall both (a) rely on 

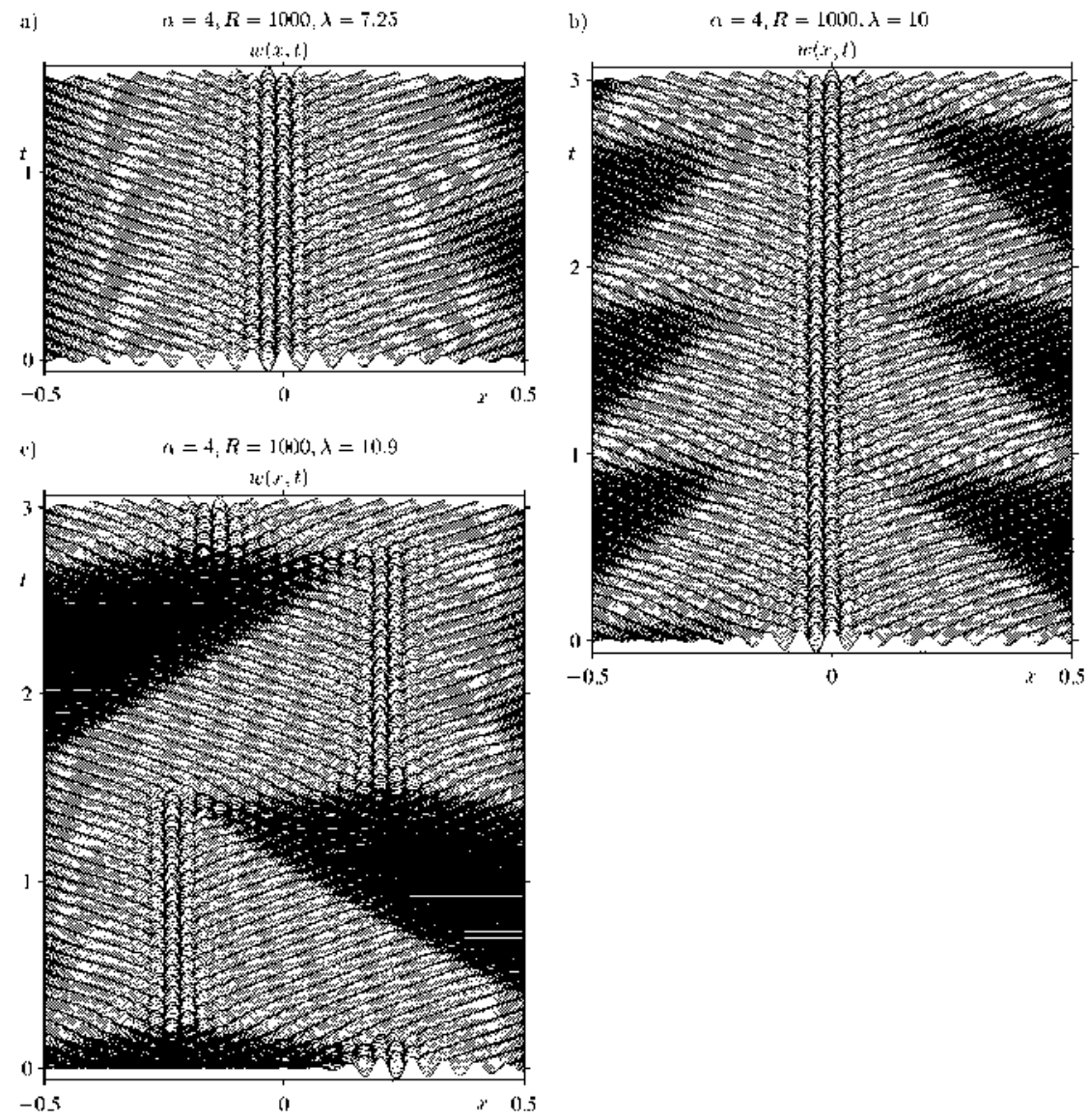

Figure 13. $x-t$ diagrams for $\lambda=7.25,10$ and 10.9 in figure 12 .

some analytical results from [4] and (b) numerically integrate the equation to obtain the associated Lyapunov exponents. Our results will allow us to conjecture that the sign of the first Lyapunov exponent coincides with that of the quantity $-\left(1+\alpha_{1} \alpha_{2}\right)$, where $\alpha_{1}$ and $\alpha_{2}$ are as defined in (1.24), independently of the basic solution (not containing intermediate scales) of (1.1), (1.2) we are perturbing. That simple condition for the appearance of the intermediate scales, namely

$$
1+\alpha_{1} \alpha_{2}<0
$$

coincides with the condition for the modulational instability of non-zero, constant-amplitude solutions of the standard complex Ginzburg-Landau equation $[2,3]$, and this coincidence may be explained as follows. When analysing the evolution of a rapidly oscillatory perturbation added to each complex amplitude, neither the convective term associated with the group velocity, nor the cross-nonlinearity associated with the counter-propagating wavetrain nor the spatiotemporal evolution of the basic solution need to play any role. But this is of course an a posteriori explanation and, in fact, we shall see that the spatio-temporal evolution of the basic solution cannot be eliminated from the equations giving the linear evolution of the intermediate scales.

When condition (5.1) holds the solutions of the lyperbolic system (1.1), (1.2) are not 
good approximations of the solutions of the amplitude equations and the complete parabolic system (1.19)-(1.23) must be numerically integrated to obtain the evolution of the counterpropagating wavetrains. This will be done in section 5.2 where it will be numerically seen that the consistency conditions (1.9) are not violated (even though equations (1.19)-(1.23) contain terms that are not of the same order) because the smallest scales, with a wavelength of the order of $L^{-1}$ in the $x$ variable (or of order unity in the original $X$ variable) are still damped out as time proceeds.

\subsection{Linear evolution of perturbations containing intermediate scales}

Let $A_{1}=A_{1}^{j}(x, t) . B_{1}=B_{1}^{j}(x, t)$ be a solution of (1.19)-(1.23) not depending on the intermediate scales, and let us look for solutions of $(1.19)-(1.23)$ of the form

$$
\begin{array}{lll}
A_{1}=A_{0}^{1}(1+U) & B_{1}=B_{0}^{1}(1+V) & \text { if } b>0 \\
A_{1}=A_{0}^{1}(1+V) & B_{1}=B_{0}^{1}(1+U) & \text { if } b<0
\end{array}
$$

where $U$ and $V$ depend on $x, t$ and the fast space and time variables

$$
\xi=x / \sqrt{\varepsilon} \quad \text { and } \quad \tau=t / \sqrt{\varepsilon}
$$

which are associated with the intermediate scales. Here $\varepsilon=O\left(L^{-1}\right)$ is as defined in (1.24). To the linear approximation, the evolution equations for $U$ and $V$ are given by

$$
\begin{gathered}
U_{\mathrm{\tau}}-U_{\xi}+\sqrt{\varepsilon}\left[\left(U_{t}-U_{x}\right)-\left(1+\mathrm{i} \alpha_{1}\right) U_{\xi \xi}+\left(1+\mathrm{i} \alpha_{2}\right) u(U+\bar{U})\right. \\
\left.+\left(\alpha+\mathrm{i} \alpha_{3}\right) v(V+\bar{V})\right]=\mathrm{HOT} \\
\begin{aligned}
& V_{\mathrm{\tau}}+V_{\xi}+\sqrt{\varepsilon} {\left[\left(V_{t}+V_{x}\right)-\left(1+\mathrm{i} \alpha_{1}\right) V_{\xi \xi}+\left(1+\mathrm{i} \alpha_{2}\right) v(V+\bar{V})\right.} \\
&\left.+\left(\alpha+\mathrm{i} \alpha_{3}\right) u(U+\bar{U})\right]=\mathrm{HOT}
\end{aligned} \\
\begin{aligned}
U=V \quad \text { at } x= \pm \frac{1}{2} \quad \text { and } \quad \xi= \pm 1 /(2 \sqrt{\varepsilon})
\end{aligned}
\end{gathered}
$$

where definitions (1.25) have been taken into account, the boundary conditions (1.22), (1.23) are not written because they will not be used below and HOT $=\mathrm{O}(\varepsilon|U|+\varepsilon|V|)$. If the perturbations $U$ and $V$ are expanded as

$$
U=U_{0}+\varepsilon^{1 / 2} U_{1}+\cdots \quad V=V_{0}+\varepsilon^{1 / 2} V_{1}+\cdots
$$

then $U_{0}$ and $V_{0}$ are readily seen to satisfy the following linear wave equations $U_{0 r}-U_{0 \xi}=$ $V_{0 \tau}+V_{0 \xi}=0$, whose solution may be found as a superposition of normal modes, of the type

$$
\left(U_{0}, V_{0}\right)=\left(U_{0}^{k}(x, t) \mathrm{e}^{\mathrm{i} k(\tau+\xi)}, V_{0}^{k}(x, t) \mathrm{e}^{\mathrm{i} k(\tau-\xi)}\right)
$$

with $0<k^{2}<\infty$. If this solution (to the $O(1)$ problem) is inserted into the equations giving $U_{1}$ and $V_{1}$ and secular terms are eliminated. that is, if $U_{1}$ and $V_{1}$ are required to be bounded in the timescale $\tau \sim \mathrm{I}$ (or, equivalently. the right-hand sides of the equations giving $U_{1}$ and $V_{1}$ are required to exhibit no resonant terms, that is, no terms depending on the fast space and time variables as $\exp [\mathrm{i} k(\tau+\xi)]$ and $\exp [\mathrm{i} k(\tau-\xi)]$ respectively) then the following equations result

$$
\begin{aligned}
& U_{0 t}^{k}-U_{0 x}^{k}=-k^{2}\left(\mathbf{I}+\mathbf{i} \alpha_{1}\right) U_{0}^{k}-\left(\mathbf{I}+\mathbf{i} \alpha_{2}\right) u\left(U_{0}^{k}+\bar{U}_{0}^{-k}\right) \\
& U_{0 t}^{-k}-U_{0 x}^{-k}=-k^{2}\left(\mathbf{1}+\mathbf{i} \alpha_{1}\right) U_{0}^{-k}-\left(\mathbf{1}+\mathrm{i} \alpha_{2}\right) u\left(U_{0}^{-k}+\bar{U}_{0}^{k}\right) \\
& V_{0 t}^{k}+V_{0 x}^{k}=-k^{2}\left(\mathbf{I}+\mathbf{i} \alpha_{1}\right) V_{0}^{k}-\left(\mathbf{I}+\mathrm{i} \alpha_{2}\right) v\left(V_{0}^{k}+\bar{V}_{0}^{-k}\right) \\
& V_{0 t}^{-k}+V_{0 x}^{-k}=-k^{2}\left(1+\mathbf{i} \alpha_{1}\right) V_{0}^{-k}-\left(1+\mathrm{i} \alpha_{2}\right) v\left(V_{0}^{-k}+\bar{V}_{0}^{k}\right)
\end{aligned}
$$


with $0<k<\infty$. These equations must be integrated with the following boundary conditions that are readily obtained from (5.5)

$$
\begin{array}{ll}
U_{0}^{ \pm k}=V_{0}^{ \pm k} \exp \left( \pm \mathbf{i} v_{k}\right) & \text { at } x=-\frac{1}{2} \\
V_{0}^{ \pm k}=U_{0}^{ \pm k} \exp \left( \pm \mathbf{i} v_{k}\right) & \text { at } x=\frac{1}{2}
\end{array}
$$

where $v_{k}$ is equal to $2 \pi$ [fractional part of $k /(2 \pi \sqrt{\varepsilon})$ ]. For convenience we shall use the following new variables

$U_{k}^{+}=\left(U_{0}^{k}+\bar{U}_{0}^{-k}\right) \exp \left[\mathbf{i} v_{k}(t+x)\right] \quad U_{k}^{-}=-\mathbf{i}\left(U_{0}^{k}-\bar{U}_{0}^{-k}\right) \exp \left[\mathbf{i} v_{k}(t+x)\right]$

$V_{k}^{+}=\left(V_{0}^{k}+\bar{V}_{0}^{-k}\right) \exp \left[\mathrm{i} v_{k}(t-x)\right] \quad V_{k}^{-}=-\mathrm{i}\left(V_{0}^{k}-\bar{V}_{0}^{-k}\right) \exp \left[\mathrm{i} v_{k}(t-x)\right]$

to rewrite $(5.7)-(5.12)$ as

$$
\begin{aligned}
& U_{k t}^{+}-U_{k x}^{+}=-\left(k^{2}+2 u\right) U_{k}^{+}+\alpha_{1} k^{2} U_{k}^{-} \\
& U_{k t}^{-}-U_{k x}^{-}=-\left(\alpha_{1} k^{2}+2 \alpha_{2} u\right) U_{k}^{+}-k^{2} U_{k}^{-} \\
& V_{k t}^{+}+V_{k x}^{+}=-\left(k^{2}+2 v\right) V_{k}^{+}+\alpha_{1} k^{2} V_{k}^{-} \\
& V_{k t}^{-}+V_{k x}^{-}=-\left(\alpha_{1} k^{2}+2 \alpha_{2} v\right) V_{k}^{+}-k^{2} V_{k}^{-} \\
& U_{k}^{ \pm}=V_{k}^{ \pm} \quad \text { at } x= \pm \frac{1}{2}
\end{aligned}
$$

with $0<k<\infty$. Note that the phase shift in the boundary conditions has been eliminated and that all coefficients are now real.

Now, by collecting the results of the formal analysis above and assuming that nonlinear terms do not change linear, exponential stability properties. we may state the following property. Let $(u, v)$ be a solution of $(1.1)-(1.3)$. If, for each $k>0$, every solution of (5.13)-(5.17) is damped out exponentially as $t \rightarrow \infty$ then the intermediate scales can be ignored and ( $u$, v) provides a good approximation of a solution of (1.19)-(1.23). If, instead, there is a positive value of $k$ and a solution of (5.13)-(5.17) such that

$$
\liminf _{|x| \leqslant 1 / 2 . t \rightarrow \infty} \mathrm{e}^{-\delta t}\left[\left|U_{k}^{+}(x, t)\right|+\left|U_{k}^{-}(x, t)\right|+\left|V_{k}^{+}(x, t)\right|+\left|V_{k}^{-}(x, t)\right|\right]>0
$$

for some $\delta>0$, then the intermediate scales cannot be ignored and the solution of (1.1)$(1.3),(u, v)$, does not provide a good approximation of (1.19)-(1.23). Note that when checking this property we may consider only real solutions of $(5.13)-(5.17)$ because. since the coefficients are real, the real and imaginary parts of any complex solution of (5.13)(5.17) also satisfy the same problem.

Now, the system (5.13)-(5.17) may be solved in close-form only if $u$ and $v$ are constant, and this can only happen if the modified reflection coefficient is equal to 1 . In this case we readily obtain that the intermediate scales can be ignored if

$$
1+\alpha_{1} \alpha_{2}>0
$$

and that they cannot be ignored if

$$
1+\alpha_{1} \alpha_{2}<0 \text {. }
$$

In the general case the system $(5.13)-(5.17)$ may still be qualitatively analysed [4, appendix $\mathrm{B}]$ to show that if $\left(5.18^{\prime}\right)$ holds then the intermediate scales cannot be ignored, while they can be ignored if either

$$
0<1+\alpha_{1} \alpha_{2}<1
$$

or

$$
1+\alpha_{1} \alpha_{2} \geqslant 1 \quad \text { and } \quad k \geqslant k_{1}
$$


for some constant $k_{1}$ depending on $(u, v)$. Note that all these properties are independent of $(u, v)$ (except for the constant $k_{1}$ ). Unfortunately we have been unable to prove that every solution of (5.13)-(5.17) is exponentially damped out if (5.18) holds, without further restrictions on the scaled-wavelength $k$, associated with the intermediate scales. but our numerical results below made us to conjecture that this property also holds.

In order to ascertain this conjecture and to quantify the incipient role of the intermediate scales we have numerically integrated (1.1)-(1.3), (5.13)-(5.17) for several values of the coefficients $\lambda, \alpha, R, \alpha_{1}$ and $\alpha_{2}$. and a set of values of the scaled wavelength $k$, and have calculated the first few Lyapunov exponents associated with (5.13)-(5.17) as usual [17], by considering several, linearly independent initial conditions and applying a reorthonormalization procedure at $t=T, 2 T, 3 T, \ldots$, for some fixed $T$. As expected, the Lyapunov exponents do not depend on the initial conditions (1.3); they depend only on the asymptotic behaviour of $(u, v)$ as $t \rightarrow \infty$. The first Lyapunov exponent associated with the intermediate scales is plotted in terms of the scaled wavelength $k$ in figure $\mathbf{1 4}$, for several values of the quantity $1+\alpha_{1} \alpha_{2}$ and several representative attractors of (1.1)-(1.3) (namely, a steady state, a limit cycle. a quasiperiodic attractor and two chaotic attractors) of the bifurcation diagrams in figures 8.10 and 12 . The transition has been checked to be always at $\mathbf{I}+\alpha_{1} \alpha_{2}=0$. and to be associated to a long-wave instability (namely, the instability of the intermediate scales first appears near $k=0$ ). That property must be taken into account when deriving a nonlinear equation giving the incipient, weakly nonlinear evolution of the intermediate scales, for $-1-\alpha_{1} \alpha_{2}$ positive and small; we do not pursue this matter any further because it is somewhat apart from the main scope of this paper. Instead, we shall only consider below several cases when $-1-\alpha_{1} \alpha_{2}$ is positive and of order unity.

\subsection{Solutions of the complex amplitude equations with intermediate scales}

Let us now consider the case when $1+\alpha_{1} \alpha_{2}<0$ and the approximation leading to the hyperbolic system (1.1)-(1.3) fails. In this case we must numerically integrate the complete problem (1.19)-(1.23). This is done below by a standard (except for the nonlinear boundary conditions (1.23) that must be handled with some care) implicit. finite difference scheme.

For the sake of brevity, and because numerics is not cheap if $\varepsilon$ is small and the problem (1.19)-(1.23) depends on so many parameters, we cannot pretend any completeness. Instead, for illustration we only consider a case when the solution without intermediate scales is as simple as possible, namely, a perfect standing wave (this requires the reflection coefficient $\rho$ to be equal to 1$)$. Then any complexity of the resulting solutions of $(1.19)-(1.23)$ is entirely due to the intermediate scales, and their role will be somehow uncovered. A piece of the large time evolution of the modulus of the rescaled complex amplitudes, $A_{1}$ and $B_{1}$, is plotted in figure 15 (plots $(a)-(c)$ ) for three sets of values of the parameters. Note that:

(a) The size of the stnictures are of the order of $\sqrt{\varepsilon}=1 / \sqrt{500}$. Smallest stnictures, of the order of $\varepsilon$ are damped out and the consistency conditions (1.9) hold. This is appreciated in figure 15 and has also been quantitatively checked through the Fourier spectra of $A_{1}$ and $B_{1}$.

(b) The structures approximately evolve with the group velocity (that equals one in the present scaling) and are purely reflected at the end-walls, as was to be expected.

(c) As dispersion $\left(\left|\alpha_{1}\right|\right)$ increases the separation between nearby solitary waves increases, while nonlinearity $\left(\left|\alpha_{2}\right|\right)$ leads to the opposite trend.

(d) The number of solitary waves travelling in opposite directions is not constant. The system sometimes exhibits creation and annihilation of waves, pairing of nearby waves and splitting. 

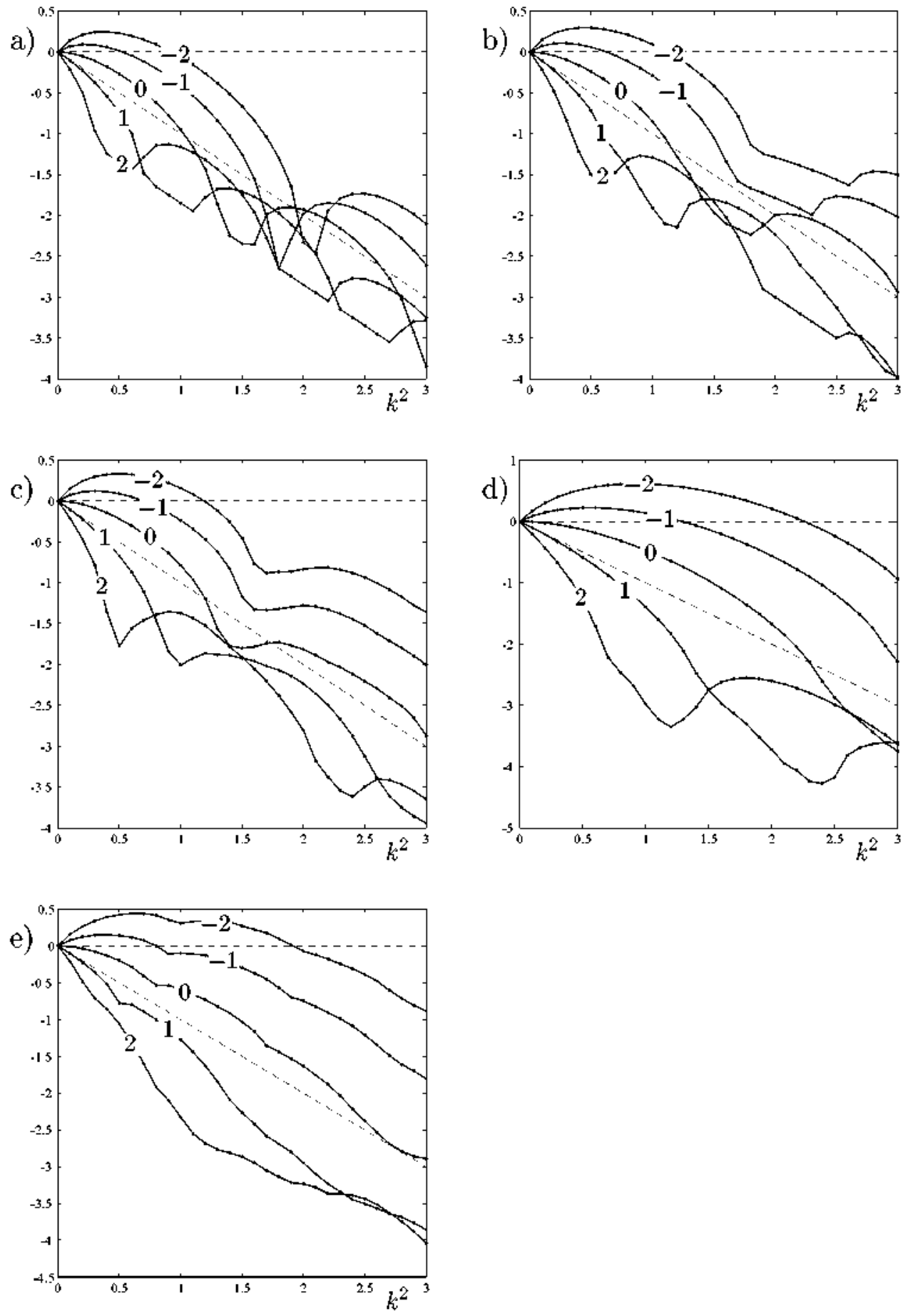

Figure 14. First Lyapunov exponents of (5.13)-(5.17) for the solutions of (1.1)-(1.3): $(a) R=$ $0.001, \alpha=2, \lambda=6$ (steady); (b) $R=1, \alpha=3, \lambda=4$ (periodic); (c) $R=0.001, \alpha=2, \lambda=10$ (chaotic); (d) $R=1000, \alpha=4, \lambda=9$ (quasiperiodic) and (e) $R=1000, \alpha=4, \lambda=11.5$ (hyperchaotic). Labels are the corresponding values of $1+\alpha_{1} \alpha_{2}$. 
(e) Both $A_{1}$ and $B_{1}$ vanish at some particular values of $x$ and $t$. This means that the total phase shift of $A_{1}$ and $B_{1}$ between the end-walls is not constant and yields dislocations in the $x-t$ diagrams when both wavetrains are plotted simultaneously and the basic wavelength, of the order of $\varepsilon$, is also considered as we do now. To this end. in figure $15(d)$ we plot the $x-t$ diagram of $|w|$. where $w$ is the state variable

$$
w=A_{1} \mathrm{e}^{\mathrm{l}(\Omega L t+k x) L}+B_{1} \mathrm{e}^{\mathrm{l}(\Omega 2 t-k x) L}+\text { c.c. }
$$

where $A_{1}$ and $B_{1}$ are as in figure 15 (c) and, for illustration, we take $\Omega L=k L=40 \pi$. That plot contains too much information and only overall properties are clearly appreciated. We just point out two of them. First, the spatial order is lost (compare with the plots in, for example figures 11 or 13 ) and, secondly, spacetime defects appear.

\section{Comparison with experiments}

Let us now discuss several experimental results concerning the oscillatory instability on the light of our theoretical results in sections 3 and 4 . We shall consider three physical problems, namely, a secondary instability of rolls in pure Rayleigh-Bénard convection, the transversal instabilities of two-dimensional thermocapillary flows and of the flow between counter-rotating cylinders. Unfortunately the degree of precision of the experimental results in the literature is not always good enough near threshold and some comparisons can only be qualitative.

\subsection{A quantitative comparison in pure Ravleigh-Bénard conection}

The oscillatory instability appears as a secondary instability on straight convective rolls at low Prandtl number, in (pure Rayleigh-Bénard convection in) a fluid layer heated from below. Here the bifurcation parameter is the Rayleigh number.

We shall consider some experimental results by Croquette and Williams [18, 19] who used pressurized Argon gas (at $60 \mathrm{~atm}$ ) between two rectangular, parallel plates, with horizontal sizes $24 d \times 31.7 d$, where $d=1 \mathrm{~mm}$ was the distance between them. The Rayleigh number was controlled quite precisely through the temperature slift between the plates, that was regulated within $0.002^{\circ} \mathrm{C}$. Their experimental results concerning the oscillatory instability in a 20-roll pattern may be summarized as follows. In terms of a critical value of the Rayleigh number, $R a^{c}$, corresponding to the first appearance of convective rolls. The oscillatory instability first appeared at $R a^{1}=3.929 R a^{c}$ via a supercritical bifurcation to a pair of wavetrains that counter-propagated along the axis of the rolls from the centre to the end-walls; the associated wavelength was well defined and independent of the Rayleigh number (eight wavelengths were always seen along the axis of the rolls). In the range $R a^{1}<R a<R a^{3}=4.283 R a^{c}$ the complex amplitudes of the counter-propagating waves were steady; the complex amplitudes were calculated by digital filtering and Fourier analysis techniques to first separate the two waves and then remove the fast spatial and temporal variations. The pattem was symmetric in $R a^{1}<R a<R a^{2}=4.12 R a^{c}$ and non-symmetric in $R a^{2}<R a<R a^{3}$, after exhibiting a supercritical symmetry-breaking bifurcation at $R a=R a^{2}$. At $R a^{3}$ they observed a Hopf bifurcation to a monoperiodic behaviour of the complex amplitudes, and the bifurcated branch exhibited a supercritical period-doubling bifurcation at $R a=R a^{4}$, with $4.366 R a^{c}<R a^{4}<4.417 R a^{c}$. For higher values of $R a$ the pattern became chaotic and still essentially one-dimensional, but when $R a$ was further increased the phase coherence from roll to roll was lost and the pattern became clearly two-dimensional (and thus out of the present analysis). 

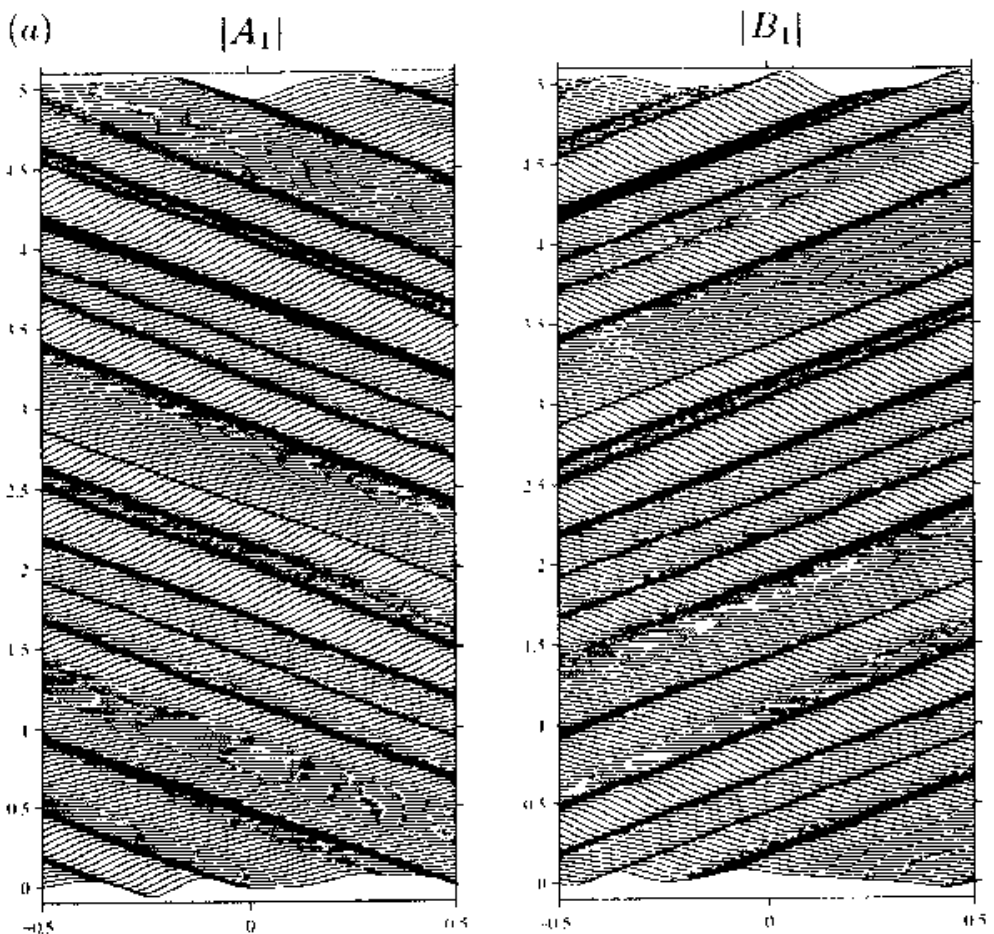

(b)

$\left|A_{1}\right|$

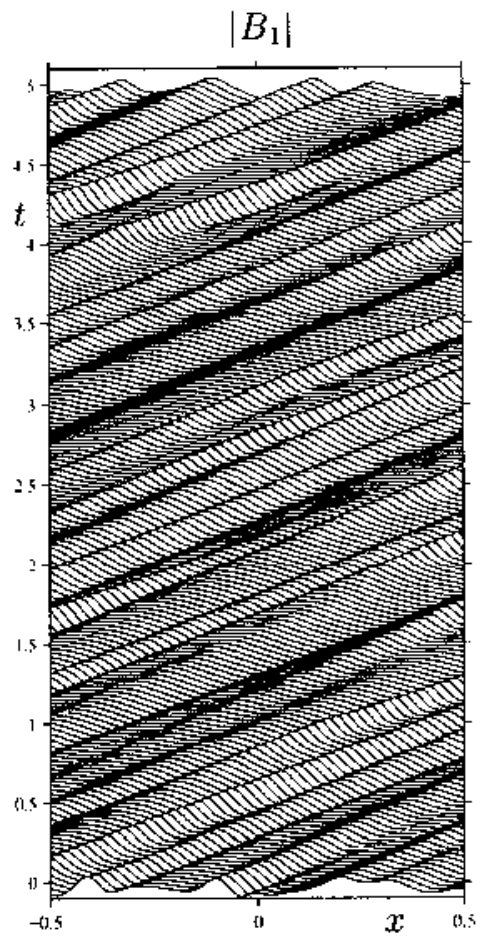

Figure 15. Solutions of the parabolic problem (1.19)-(1.23) for $\varepsilon=\frac{1}{300}, b>0, \rho=1, \lambda=$ $1.5, \alpha_{x}=3, \alpha_{3}=0, \alpha_{4}=0$ and: $(a) \alpha_{1}=1, \alpha_{2}=-3,(b) \alpha_{1}=1, \alpha_{2}=-12$ and (c) $\alpha_{1}=6, \alpha_{2}=-3$. The $x-1$ diagram of the state variable $(5.19)$ for the case in $(c)$ is plotted in $(d)$. 

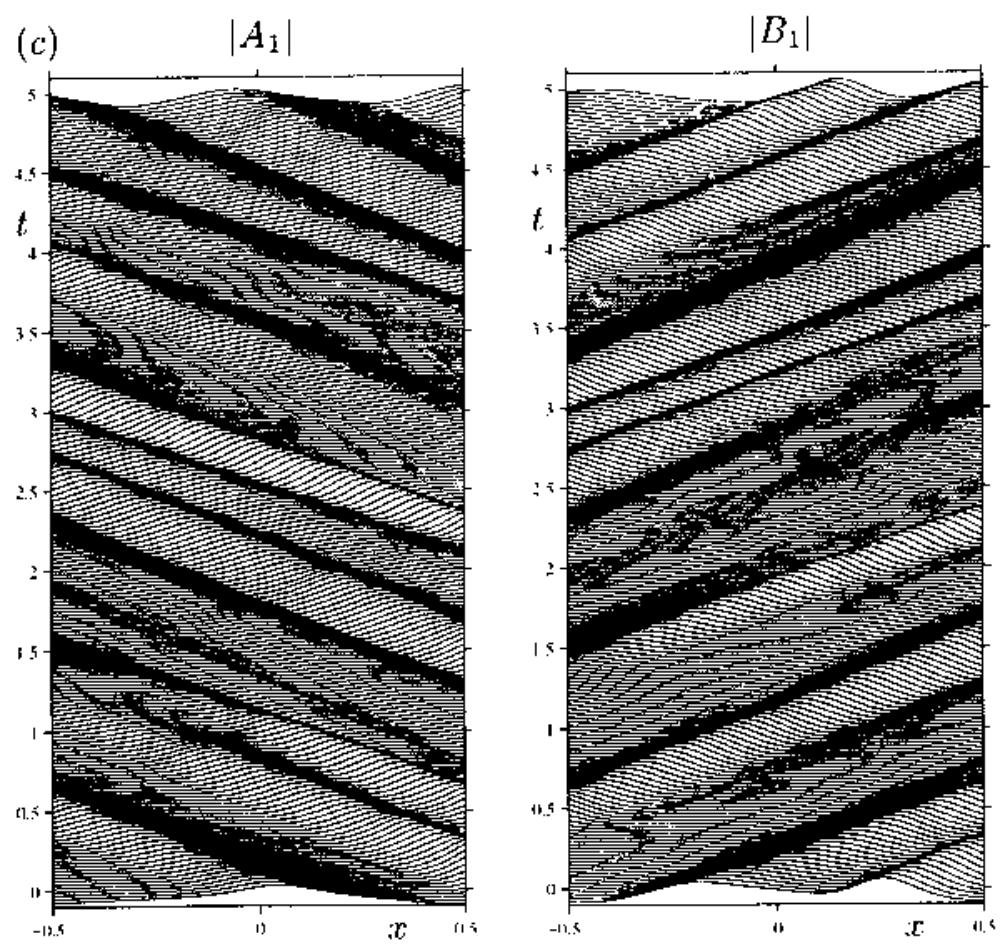

Figure 15. (Continued)

Now. the end-walls were clearly absorbing and the group velocity is known to be positive for this experiment [18-20]. Thus. according to equation (1.25) above. the modified reflection coefficient $R=\rho^{2}$ is smaller than 1 . In addition, the coupling coefficient $\alpha$ is known to be approximately equal to $2[21]$ and quantitative comparison with our results in sections 2 and 3 would be at hand if the modified reflection coefficient $R$ were estimated. But before proceeding further, note that the critical Rayleigh number associated with the onset of the oscillatory instability in infinite domains, $R a^{\circ}$ (corresponding to the value $\lambda=0$ of our bifurcation parameter) is not known and, in addition, there is an unknown factor between $R a-R a^{0}$ and $\lambda$. Then we only may compare quotients of differences of bifurcation values such as

$$
\left(R a^{3}-R a^{1}\right) /\left(R a^{2}-R a^{1}\right)=\left(\lambda_{\mathrm{H}}-\lambda_{C}\right) /\left(\lambda_{\mathrm{SB}}-\lambda_{C}\right) .
$$

The authors of $[18,19]$ did some experiments to obtain the linear dispersion relation and the critical Rayleigh number in infinite domains to obtain (through the shift $R a^{1}-R a^{0}$ ) the reflection coefficient $\rho$, that they estimated it to be $\rho=0.183$. With that value of $\rho$, $R=\rho^{2}=0.0335$, and $\alpha=2$ our analysis above predicts a value of the quotient (6.1). 2.07 , which compares reasonably well with the measured value, 1.85 ; but a glance at the complex amplitudes profiles in [19, figures 10 and 18] shows that $\rho$ must be much smaller. It might be, in fact, as small as 0.03 , and thus $R=0.001=\rho^{2}$ may be a good choice for the modified reflection coefficient. That value of $R$ (with $\alpha=2$ ) provides a much better estimate of the quotient (6.1), namely, 1.863; in fact, as $R \rightarrow 0$ the quotient (6.1) converges to a constant (see figure 6), precisely. 1.82, that compares also well with the measured value. If we take $R=0.001$ then the calculated bifurcation diagram is as given in figures $4(a)$ 


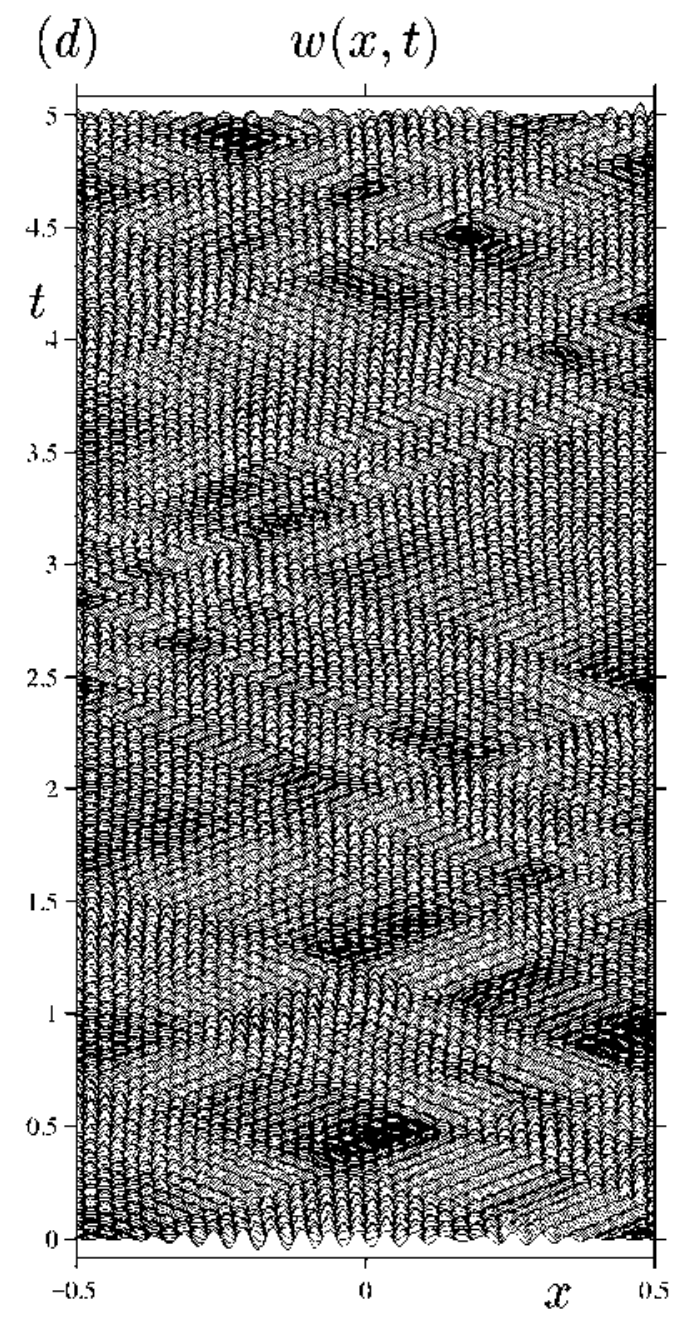

Figure 15. (Continued)

and 8 and exhibits the same bifurcations as in the experiment described above. In order to obtain a more accurate value of $R$ we compare the measured profiles in [19, figures $10(a)$ and $(b)$ ], corresponding to a steady symmetric and steady non-symmetric solutions, with our theoretically obtained ones. The experimental profiles correspond to the ratios

$$
\left(R a-R a^{1}\right) /\left(R a^{2}-R a^{1}\right) \simeq 0.56,1.53
$$

respectively, and the associated values of our bifurcation parameter $\lambda$ are readily obtained from the relation

$$
\lambda=\lambda_{\mathrm{C}}+\left(\lambda_{\mathrm{SB}}-\lambda_{\mathrm{C}}\right)\left(R a-R a^{1}\right) /\left(R a^{2}-R a^{1}\right) .
$$

Then the theoretical profiles depend only on $R=\rho^{2}$ (recall that $\alpha=2$ is fixed) and on an unknown scaling factor that is not given in [19]. By using a least squares method we find that the best approximation occurs for $R=0.006$, and the fitting of the profiles is quite good (see figure 16). For this value of $R$, the quotient (6.1) is readily obtained from figure 6 to be equal to 1.91. and also compares well with the experimental value, 1.85. Also, 

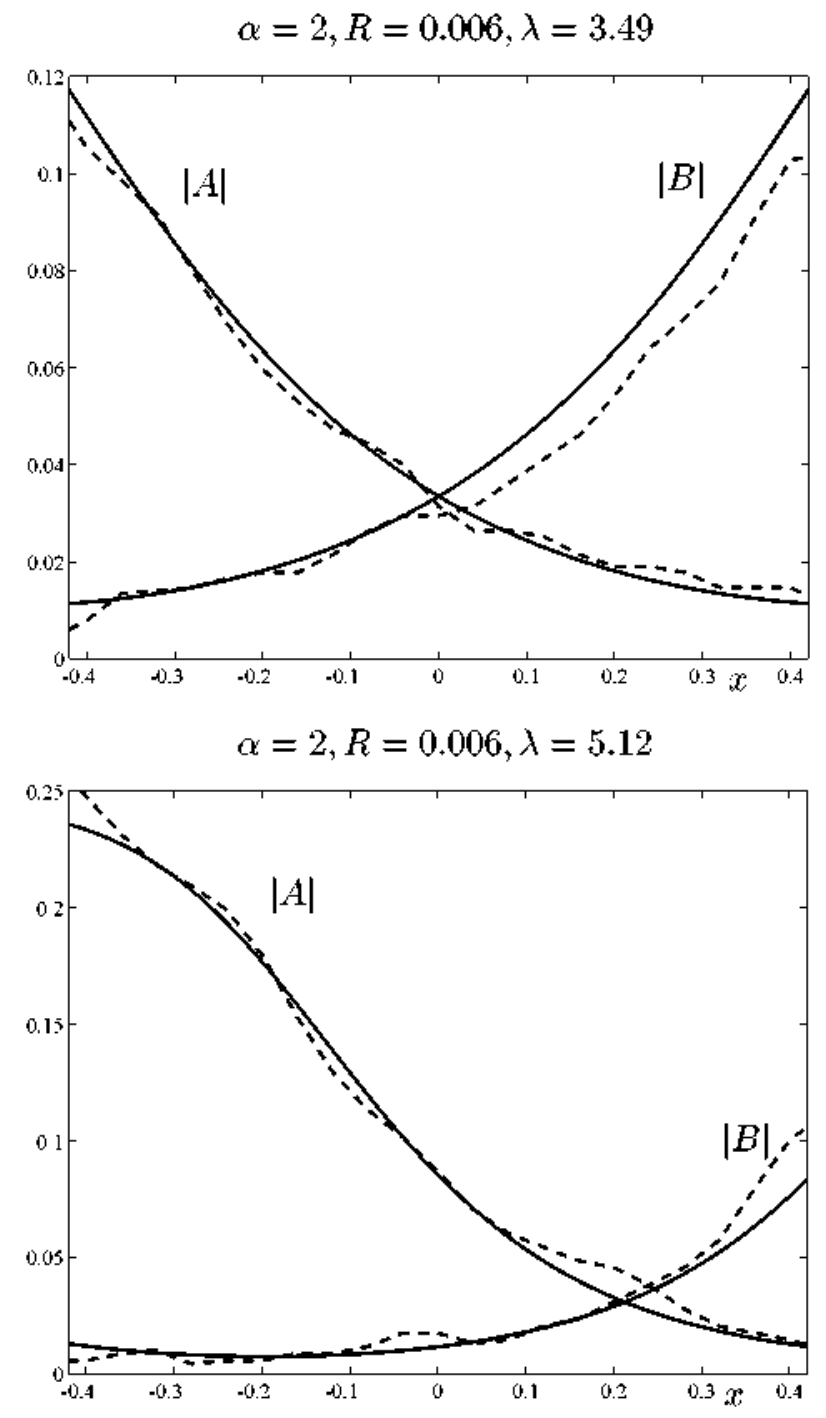

Figure 16. Comparison with experimental results from [19]. Broken curves: experimental profiles, full curves: solutions of $(1.1)-(1.3)$ for the indicated values of the parameters.

the theoretical bifurcation diagram (plotted in figure 17) exhibits the same main transitions as those reported in [19]: namely, the primary bifurcation. a symmetry breaking, a Hopf bifurcation, a period doubling sequence and a transition to a chaotic dynamics.

To conclude, we have selected the value $R=0.006$ for comparison with experimental results. With this value of $R$ the theoretical bifurcation diagram exhibits the same transitions as those observed experimentally; the associated values of the bifurcation parameter and the solutions compare reasonably well with experiments. But we do not claim any precision in the estimated value of the modified reflection coefficient $(R=0.001$ gives equally acceptable results) because at these small values of $R$ the quotient (6.1) is quite insensitive to changes in $R$. In addition: (a) we do not know how good the approximation $\alpha=2$ is: (b) the large parameter $L$ is only of the order of 10 and thus higher-order terms. not considered in 


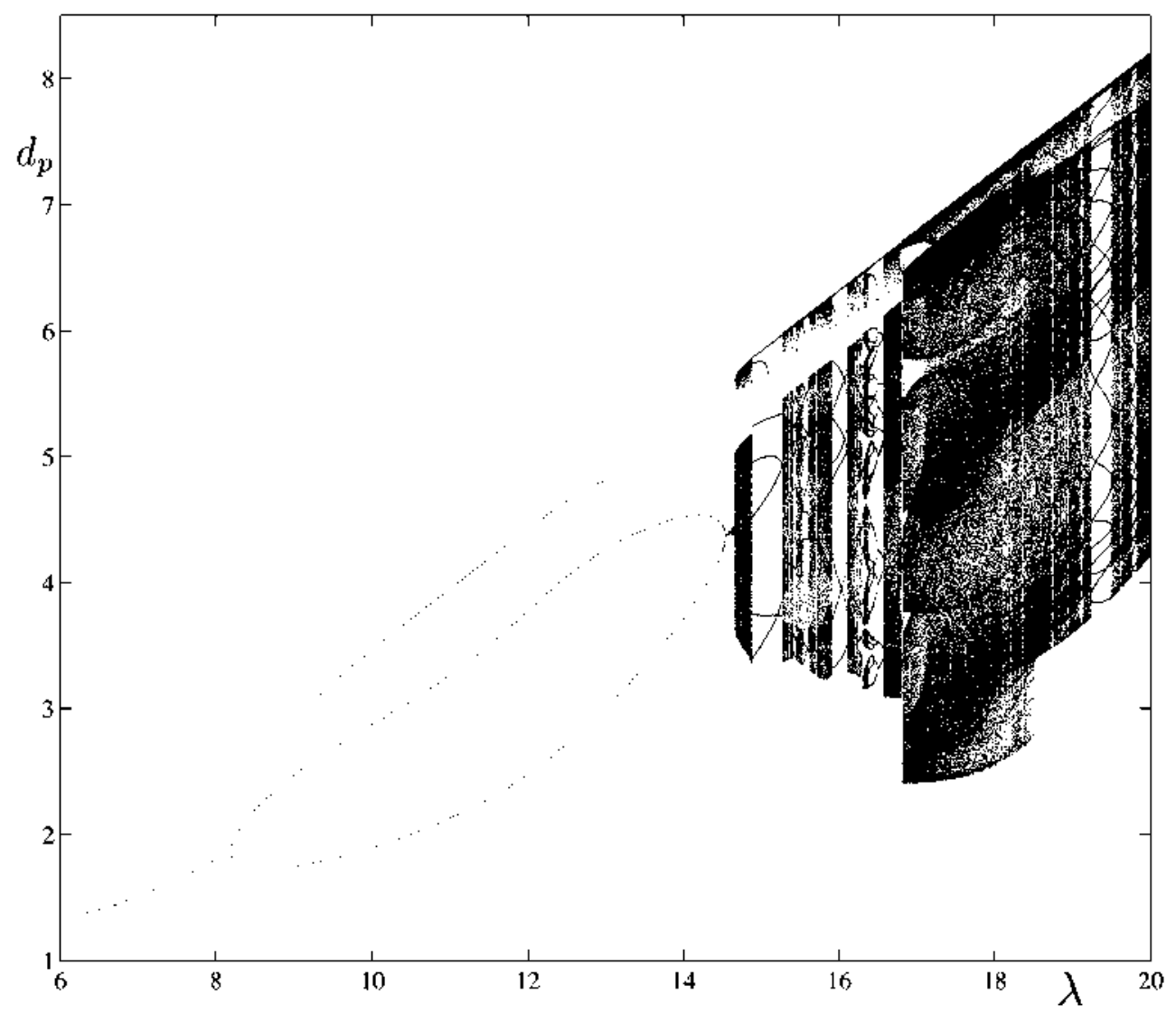

Figure 17. Poincare sections of the solutions of (1.1)-(1.3) for $\alpha=2$ and $R=0.006$.

sections 2 and 3 could have a non-negligible effect in quantitative comparisons: and (c) our results must worsen as the bifurcation parameter grows (essentially, their validity requires $\lambda / L \ll 1$ ). In connection with the last remark, note that the authors of [18. 19] reported a more complex two-dimensional behaviour. suggesting a fully nonlinear evolution when $\lambda$ is significantly increased. Finally, the wavelength of the counter-propagating wavetrains is essentially independent of the bifurcation parameter in the experiment, as predicted by our theory in sections 2 and 3 . This would not be true if smaller, intermediate scales were present, as seen in section $\mathbf{5}$, where we have conjectured the condition $\mathbf{1}+\alpha_{1} \alpha_{2}>0$ (with $\alpha_{1}$ and $\alpha_{2}$ as defined in (1.24)) as necessary and sufficient for the small scales to be inhibited. And in fact, the constants $\alpha_{1}$ and $\alpha_{2}$ are predicted in [19] to be $\alpha_{1} \simeq-0.86$ and $\alpha_{2} \simeq-1.15$; thus the above-mentioned condition holds.

\subsection{Qualitative comparisons}

The remaining comparisons here are necessarily qualitative because of the lack of precise quantitative experimental results near the threshold of the oscillatory instability.

(a) Oscillatory thermocapillary flows have received great attention in the literature due to their interest in float-zone crystal growth: the associated experiments are generally performed in cylindrical geometries and involve complex spatio-temporal two- and three-dimensional 
patterns [22]. Some simpler one-dimensional patterns have also been obtained in simpler geometries that are considered now.

(a.1) Daviaud and Vince [23] used a container $20 \mathrm{~cm}$ long and $1 \mathrm{~cm}$ wide filled with silicone oil of viscosity $v=0.0065$ Stokes and Prandtl number $\operatorname{Pr}=10$. up to a height $h$ ranging from 0 to $10 \mathrm{~mm}$. They applied a horizontal temperature gradient along the smaller dimension of the container by fixing the temperatures of the walls. In the range $0<h<2.8 \mathrm{~mm}$ they increased the temperature difference $\Delta T$ and observed that there was a threshold value (depending on $h)$ such that if $0<\Delta T<(\Delta T)_{c}$ then only a steady, two-dimensional thermocapillary flow was observed, while for $\Delta T>(\Delta T)$ c a pair of wavetrains appeared that counter-propagated perpendicularly to the temperature gradient from a source to the end-walls. From the results reported in [23] we can see that the endwalls are absorbing and the phase velocity of the waves is fairly independent of $\Delta T$ near the threshold if $h \geqslant 1.42 \mathrm{~mm}$, while a clear dependence is observed for $h \leqslant 1.1 \mathrm{~mm}$. The patterns were steady and qualitatively similar to those in the first two plots in figure $5(a)$, suggesting a value of the coupling coefficient $\alpha$ such that $\alpha<1$ : but spacetime dislocations were reported that could be due to intermediate scales if they appear close to the threshold or to fully nonlinear effects, not included in our theory, otherwise. Unfortunately a precise description is not given near the threshold and direct comparison with our results above is not possible.

(a.2) A somewhat similar experiment was performed by Vince and Dubois [24] (see also [25]) who created a symmetric, horizontal temperature gradient in the cross section of the container by setting a long, hot wire just below the free surface, the heat power being electrically supplied to the wire. The observations were presented in terms of two parameters, the distance $h$ from the wire to the free surface and the heat power $Q$ supplied to the wire. For a given value of $h$ a threshold value $Q_{c}$ (depending on $h$ ) was observed such that for $Q<Q_{c}$ only a two-dimensional steady flow was observed. while for $Q>Q_{c}$ a pair of counter-propagating waves appeared parallel to the wire. Unfortunately no precise description is given near the threshold and quantitative comparison is again not possible. The main observed feature was that for $h$ smaller than a critical value $h_{0}$ the propagative pattern (consisting of a source of waves inside the container and two sinks near the endwalls) was quite ordered, while for $h>h_{0}$ the pattern exhibited a continuous wavenumber distribution and spacetime dislocations. Then we are led to conjecture that this second regime corresponds to the appearance of intermediate scales; note that a different conjecture to explain this regime, associated with noise amplification, was made in [26]

(b) The oscillatory instability is also the pattern-formation mechanism in experiments dealing with for example Taylor-Conette convection between counter-rotating cylinders [27]. isothermal double-diffusive convection [28] or double-diffusive convection [29]. But although some of the $x-t$ diagrams in this paper are qualitatively similar to the experimentally observed ones, a precise comparison is not possible because of the lack of appropriate experimental results near the onset.

\section{Concluding remarks}

We have considered a hyperbolic system that applies near the onset of the oscillatory instability in appropriately large spatial domains when only the scales of the order of the spatial domain come into play. That system is real and depends only on the bifurcation parameter $\lambda$, the coefficient of the cross-nonlinearity $\alpha$ and a modified reflection coefficient $R$. If $-1<\alpha \leqslant 1$, the system exhibits only symmetric steady states, which are always globally, asymptotically stable. If $\alpha>1$ instead, the system always exhibits, at least. two 
secondary bifurcations, a symmetry-breaking and Hopf bifurcation; for larger values of the bifurcation parameter the bifurcation diagram is much more complex, and strongly depends on the specific values of $R$ and $\alpha$.

When smaller, intermediate scales come into play. the hyperbolic system no longer applies. A linear system has been derived for the incipient evolution of the scales, and a simple (necessary and sufficient) condition has been conjectured for the intermediate scales to come into play. This condition (seems to apply with great generality and) coincides with that giving the onset of the modulational instability of the simplest, non-trivial standing wave solutions that appear when spatial periodicity is imposed. The complete set of amplitude equations was numerically integrated in a case when the intermediate scales cannot be ignored.

Finally, a comparison with experiments was performed in the only case when we were able to find precise, quantitative experimental results near the onset of the instability. Some comments on qualitative comparisons were made in other cases.

In addition to the comments made above on the hyperbolic model (section 2) and on specific results (sections 3-5), some additional remarks are now made for convenience.

(a) Our theory above (and in [4]) is based on rational (rather than phenomenological) approximations and, consequently, it is expected to explain both qualitatively and quantitatively. experimental results provided that the assumptions justifying the approximations hold. Some of those assumptions have already been commented in sections 1 and 2, and some others are considered now. All of them should be considered when comparing with experiments.

(b) We assumed that the group velocity is of order unity. If it is small, then a different, codimension-two limit must be considered; see $[30,26]$ for analyses of this limit in the stationary and non-stationary cases, and [5] for further references. The wavenumberselection phenomenon mentioned in $[26,30]$ comes from particular source and sink solutions that are only acceptable when diffusion, dispersion and convection are of the same order. In our case. instead. stable sources or sinks (depending on the sign of the group velocity and on whether the reflection coefficient is smaller or larger than I, see our discussion in remark (e) of section 2) appear only as solutions of the hyperbolic system, as a balance of convection, nonlinearity and the effect of the end-walls, when the intermediate scales are inlibited.

(c) Our theory is one-dimensional as the experimental results are quite frequently, sometimes (but not always) due to anisotropy properties. The extension of our theory to cover the multidimensional case is expected to be highly non-trivial, with the spatial isotropy properties and the shape of the domain playing an essential role.

(d) Our theory is asymptotic and should apply at least for sufficienty small values of the parameters and variables that are assumed to be small. In practice. small numbers are just small, not 'sufficiently small'. But, as any asymptotic theory, that presented here should apply provided that the neglected terms are in fact small compared with the considered ones, and this is expected to apply quite frequently in practice near the onset of the instability (that is, for not too large values of the scaled bifurcation parameter $\lambda$ ).

(e) Our theory assumes that the primary bifurcation is supercritical and, according to several comments in sections $1-4$, this requires three conditions on the coefficients of the amplitude equations (1.6), (1.7)

$$
\operatorname{Re} c>0 \quad \operatorname{Re} e_{1}>0 \quad \operatorname{Re}\left(e_{1}+e_{2}\right)>0
$$

where Re stands for the real part. If one of these assumptions fails then a fully nonlinear analysis should be applied except in the non-generic case when subcriticality is weak, 
namely, when the term with the wrong sign is small enough and can be balanced with a higher-order term whose coefficient has the right sign to prevent blowing-up in the weakly nonlinear description. Note that this condition is concerned with relative orders of magnitude of. at least. two terms in the amplitude equations. For instance, if $\operatorname{Re} e_{1}<0$. then the validity of a weakly nonlinear description does not require just that $\left|\operatorname{Re} e_{1}\right| \ll \mathbf{I}$, but rather it requires that $\left|\operatorname{Re} e_{1}\right| \ll\left|\operatorname{Re} e_{1}^{\prime}\right|$, where $e_{1}^{\prime}$ is the coefficient of the quintic terms $\left(A|A|^{4}\right.$ and $\left.B|B|^{4}\right)$ that should be added in equations (1.6) and (1.7) to prevent blowing up.

(f) We have not considered annular domains. that is. periodic boundary conditions. As explained in [4, section 6], the extension of our theory to this case is quite straightforward.

\section{Acknowledgments}

This research was supported by DGICYT and the EEC Program on Human Capital and Mobility, under grants PB-94-0416 and CHRX-CT-93-0413.

\section{Appendix. Closed-form solutions of (3.1)-(3.3)}

If $\alpha=-1$ and $\lambda=-(\log R) / 2$ then (3.1)-(3.3) have the one-parameter family of solutions

$$
u_{s}(x)=v_{s}(-x)=(\log R) R^{x} / 2\left(c-R^{x}-R^{-x}\right) \quad \text { if } R \neq 1
$$

where the parameter $c$ varies in the interval $-\infty<c<2$ if $R<1$, and in the interval $R^{1 / 2}+R^{-1 / 2}<c<\infty$ if $R>1$. If $R=1$, then

$$
u_{s}(x)=v_{s}(x)=c
$$

with the parameter $c$ such that $0<c<\infty$. If $\alpha=-I$ and $\lambda \neq-(\log R) / 2$, then (3.1)-(3.3) have no positive solution.

If $\alpha=0$ and $\lambda>-(\log R) / 2$, then (3.1)-(3.3) have a unique solution, that is given by

$$
u_{s}(x)=v_{s}(-x)=\lambda\left(R \mathrm{e}^{\lambda}-\mathrm{e}^{-\lambda}\right) /\left[R \mathrm{e}^{\lambda}-\mathrm{e}^{-\lambda}-(R-1) \mathrm{e}^{2 \lambda . x}\right]
$$

if $\lambda \neq 0$, while if $\lambda=0$ then

$$
u_{x}(x)=v_{s}(-x)=(R-1) /[R+1-2 x(R-1)] .
$$

If $\alpha=0$ and $\lambda>-(\log R) / 2$, then (3.1)-(3.3) have no positive solution.

If $\alpha=1$ and $\lambda<-(\log R) / 2$ then (3.1) and (3.2) have a unique solution, that is written in terms of the parameter $\delta$ as follows. If $R<\mathbf{I}$ then

$\lambda=f(\delta) \operatorname{coth}(2 \delta) \quad u_{s}(x)=v_{s}(-x)=[\lambda-f(\delta) \tanh (f(\delta) x+\delta)] / 2$

for $\tanh ^{-1}((\mathbf{1}-\sqrt{R}) /(\mathbf{1}+\sqrt{R}))<\delta<\infty$, where

$$
f(\delta)=2 \tanh ^{-1}[(\mathbf{I}-\sqrt{R}) /(\mathbf{I}+\sqrt{R}) \tanh \delta] .
$$

If $R>1$ and $-(\log R) / 2<\lambda<-(\sqrt{R}-1) /(\sqrt{R}+1)$, then

$\lambda=-g(\delta) \operatorname{coth}(2 \delta) \quad u_{s}(x)=v_{s}(-x)=[\lambda+g(\delta) \operatorname{coth}(\delta-g(\delta) x)] / 2$

for $0<\delta<\infty$, where

$$
g(\delta)=2 \tanh ^{-1}[(\sqrt{R}-1) \tanh \delta /(\sqrt{R}+1)] .
$$

If $R>1$ and $\lambda=-(\sqrt{R}-1) /(\sqrt{R}+1)$, then

$u_{s}(x)=v_{s}(-x)=[(\sqrt{R}+1) / 2(\sqrt{R}-1)-x]^{-1}-(\sqrt{R}-1) /(\sqrt{R}+1)$. 
If $R>1$ and $\lambda>-(\sqrt{R}-1) /(\sqrt{R}+1)$ then

$\lambda=-h(\delta) \cot (2 \delta) \quad u_{s}(x)=v_{s}(-x)=[\lambda+h(\delta) \cot (\delta-h(\delta) x)] / 2$

for $0<\delta<\pi / 2$, where

$$
h(\delta)=2 \tan ^{-1}[(\sqrt{R}-1) \tan \delta /(\sqrt{R}+1)] .
$$

If $R=1$, then

$$
u_{s}(x)=v_{s}(-x)=\lambda / 2 \text {. }
$$

If $\alpha=\mathrm{I}$ and $\lambda \leqslant-(\log R) / 2$ then (3.1)-(3.3) have no positive solution.

\section{References}

[1] Courant R and Hilbert D 1962 Methods of Mathematical Physics vol II (New York: Interseience)

[2] Coullet $\mathrm{P}$, Fauve $\mathrm{S}$ and Tirapegui E 1985 Large scale instability of nonlinear standing waves $J$. Physigue Lett. 46 787-91

[3] Cross M C 1986 Travelling and standing waves in binary-thuid convection in finite geometries Phys. Rev. Lett. $572935-8$

[4] Martel C and Vega J M 1996 Finite size effects near the onset of the oscillatory instability Nonimeanty 9 $1129-71$

[5] Cross M C and Hohenberg P C 1993 Pattern formation outside of equilibriun Rev. Mod. Phys. 65 851-1112

[6] Clikwendu S C and Kevorkian J 1972 A perturbation method for hyperbolic equations with small nonlinearities SIAM J. Appl. Math. 22 235-58

[7] Knobloch E and De Luca J 1990 Anuplitude ejuations for travelling wave convection Nontinearity $3575-80$

[8] Alvarez-Pereira C and Vega J M 1992 On the pulsating instability of two-dimensional tlames Eur. J. Appl. Math. $355-73$

[9] Vega J M 1993 On the amplitude equations arising at the onset of the oscillatory instability in pattern formation SLAM J. Woth. Anol. 24 603-17

[10] Duan J, van Ly H and Titi E S 1994/5 The effect of non-local interactions on the dyuanics of the GinzburgLandau equation Institht Mittag-Leffer Tech. Report 31

[11] Knobloch E and Gibbon J D 1991 Coupled NLS equations for counter propagating waves in systems with reflection symmetry Phys. Lett. A 154 353-6

[12] Marte] C 1995 La Inestabilidad Oscilatoria y sus Aplicaciones en Mecánica de Fluidos y Combustión Doctoral Thesis Univeridad Politécnica de Madrid, Madrid

[13] Martel C and Vega J M 1997 A nonlocal amplitude equation that applies at the oscillatory instability in finite geometries, in preparation

[14] Martel C and Vega J M 1997 Global stability properties of a hyperbolic system arising in pattern formation Nonlin. Anal. TMA 29 439-60

[15] Tagg R, Edwards W S and Swinney H L 1990 Convective versus absolute instability in flow between counter-rotating cylinders $P$ hys. Rev. A 42 831-7

[16] Keller H B 1987 Lectures on Numerical Methods in Bifurcation Problems fata Institute of Fundamental Research Bombay) (Berlin: Springer)

[17] Wolf A, Swift J B, Swinney H L and Vastano J A 1985 Determining Lyapunov exponents from a time series Physica D 16 285-317

[18] Croquette $V$ and Willians H 1989 Nonlinear competition between waves on convective rolls Phys. Rev. A $392765-8$

[19] Croquette $\mathrm{V}$ and Williams $\mathrm{H} 1989$ Nonlinear waves of the oscillatory instability on finite convective rolls Physica D 37 300-14

[20] Clever R M and Busse F H 1974 Transition to time-dependent convection J. Fladd Mech. 65 625-45

[21] Fauve S, Bolton E W and Brachet M E 1987 Nonlinear oscillatory convection: A quantitative phase dynamics approach Physica D 29 202-14

[22] Preiser F, Schwabe D and Sharman A 1983 Steady an oscillatory thermocapillary convection in liquid colunus with free cylindrical surfaces $J$. Fluid Mech. $126545-67$

[23] Daviaud F and Vince J M 1993 Travelling waves in a fluid layer subjected to a horizontal temperature gradient Phys. Rew. E 48 4432-6

[24] Vince J M and Dubois M 1992 Hot wire below the free surface of a liquid: Structural and dynamical properties of a secondary instability Europhys Lett. 20 505-10 
[25] Dubois M, Daviaud F, Rosin O and Bergé $P 1992$ Travelling waves in pure fluids locally heated along wires Physica D 61 140-6

[26] Coullet P, Frisch T and Plaza F 1993 Sources and sinks in wave patterns Physica D 62 75-9

[27] Andereck C D. Liu S S and Swinney H L 1986 Flow regimes in a circular Couette system with independently rotating cylinders $J$. Fiuid Mech. $164155-83$

[28] Predtechensky A A, McCormick W D. Swift J B. Noszticzius Z and Swinney H L 1994 Onset of travelling waves in isothermal double diffusive convection Phys. Rev. Lett. 72 218-21

[29] Bensimon D, Kolodner P, Surko C M, Williams $H$ and Croquette V 1990 Competing and coexisting dynamical states of travelling-wave convection in an annulus $J$. Fiud Mech. $217441-67$

[30] Hohenberg P C. Kramer L and Riecke H 1985 Effects of boundaries on one-dimensional reaction-diffusion equations near threshold Physico D 15 402-20 